\title{
Li depletion in solar analogues with exoplanets
}

\section{Extending the sample $e^{\star \star \star}$}

\author{
E. Delgado Mena ${ }^{1}$, G. Israelian ${ }^{2,3}$, J. I. González Hernández ${ }^{2,3}$, S. G. Sousa ${ }^{1,2,4}$, A. Mortier ${ }^{1,4}$, N. C. Santos ${ }^{1,4}$, \\ V. Zh. Adibekyan ${ }^{1}$, J. Fernandes ${ }^{5}$, R. Rebolo ${ }^{2,3,6}$, S. Udry $^{7}$, and M. Mayor ${ }^{7}$ \\ ${ }^{1}$ Centro de Astrofísica, Universidade do Porto, Rua das Estrelas, 4150-762 Porto, Portugal \\ e-mail: Elisa.Delgado@astro.up.pt \\ 2 Instituto de Astrofísica de Canarias, C/ Via Lactea s/n, 38200 La Laguna, Tenerife, Spain \\ 3 Departamento de Astrofísica, Universidad de La Laguna, 38205 La Laguna, Tenerife, Spain \\ 4 Departamento de Física e Astronomia, Faculdade de Ciências, Universidade do Porto, 4169-007 Porto, Portugal \\ 5 CGUC, Department of Mathematics and Astronomical Observatory, University of Coimbra, 3049 Coimbra, Portugal \\ ${ }^{6}$ Consejo Superior de Investigaciones Científicas, CSIC, Spain \\ 7 Observatoire de Genève, Université de Genève, 51 ch. des Maillettes, 1290 Sauverny, Switzerland
}

Received 18 March 2013 / Accepted 25 November 2013

\begin{abstract}
Aims. We want to study the effects of the formation of planets and planetary systems on the atmospheric Li abundance of planet host stars.

Methods. In this work we present new determinations of lithium abundances for 326 main sequence stars with and without planets in the $T_{\text {eff }}$ range $5600-5900 \mathrm{~K}$. The 277 stars come from the HARPS sample, the remaining targets were observed with a variety of high-resolution spectrographs.

Results. We confirm significant differences in the Li distribution of solar twins $\left(T_{\text {eff }}=T_{\odot} \pm 80 \mathrm{~K}, \log g=\log g_{\odot} \pm 0.2\right.$ and $[\mathrm{Fe} / \mathrm{H}]=$ $\left.[\mathrm{Fe} / \mathrm{H}]_{\odot} \pm 0.2\right)$ : the full sample of planet host stars (22) shows Li average values lower than "single" stars with no detected planets (60). If we focus on subsamples with narrower ranges in metallicity and age, we observe indications of a similar result though it is not so clear for some of the subsamples. Furthermore, we compare the observed spectra of several couples of stars with very similar parameters that show differences in Li abundances up to 1.6 dex. Therefore we show that neither age, mass, nor metallicity of a parent star is the only cause for enhanced Li depletion in solar analogues.

Conclusions. We conclude that another variable must account for that difference and suggest that this could be the presence of planets that causes additional rotationally induced mixing in the external layers of planet host stars. Moreover, we find indications that the amount of depletion of $\mathrm{Li}$ in planet-host solar-type stars is higher when the planets are more massive than Jupiter.
\end{abstract}

Key words. stars: abundances - stars: fundamental parameters - planetary systems - planets and satellites: formation stars: evolution

\section{Introduction}

The study of extrasolar planets has been an exciting field of astrophysics for more than 15 years. More than 1000 planets are known in almost 800 planetary systems (Encyclopaedia of Extrasolar Planets, Schneider et al. 2011). Photospheric abundances of planet host stars are key to understanding the role of the metallicity of protoplanetary clouds in the formation of planets and to determining how the formation of planets may affect the structure and evolution of the parent stars.

* Based on observations collected at the La Silla Observatory, ESO (Chile), with the HARPS spectrograph at the $3.6 \mathrm{~m}$ ESO telescope, with CORALIE spectrograph at the $1.2 \mathrm{~m}$ Euler Swiss telescope and with the FEROS spectrograph at the $1.52 \mathrm{~m}$ ESO telescope; at the Paranal Observatory, ESO (Chile), using the UVES spectrograph at the VLT/UT2 Kueyen telescope, and with the FIES, SARG, and UES spectrographs at the $2.5 \mathrm{~m}$ NOT, the $3.6 \mathrm{~m} \mathrm{TNG}$ and the $4.2 \mathrm{WHT}$, respectively, operated on the island of La Palma in the Spanish Observatorio del Roque de los Muchachos.

$\star \star$ Table 6 is available in electronic form at http://www . aanda.org
Many studies (e.g. Gonzalez 1997; Santos et al. 2004, 2005; Fischer \& Valenti 2005; Sousa et al. 2008) have shown the significant metallicity excess of the planet-host sample compared with the sample of stars without known giant planets, suggesting a possible clue to a formation scenario. However, this metallicity excess does not seem to appear in stars which only host Neptunians and Earth-like planets (Udry \& Santos 2007; Sousa et al. 2008, 2011b). These stars have metallicities lower on average than in stars with Jupiters, and more similar to the $[\mathrm{Fe} / \mathrm{H}]$ distribution of stars without detected planets. Interestingly, Adibekyan et al. (2012a,b) have recently shown that even low-mass planet hosts with $[\mathrm{Fe} / \mathrm{H}]<-0.2$ present enhanced abundances of $\alpha$ elements and suggested that a minimum quantity of refractory material is required to form planets when the amount of $\mathrm{Fe}$ is low.

King et al. (1997) reported a difference in the $\mathrm{Li}$ abundances between the components of the binary 16 Cyg, suggesting that the presence of a planet might be responsible for the lower Li found in its host star. Using a larger sample of stars in the $T_{\text {eff }}$ range $5600-5850 \mathrm{~K}$ with detected planets, Gonzalez \& Laws (2000) and Israelian et al. (2004) show that 
exoplanets hosts are significantly more Li-depleted than stars without detected planets (hereafter called single $\operatorname{stars}^{1}$ ). This result was also supported by Takeda \& Kawanomoto (2005), Chen \& Zhao (2006), and Gonzalez (2008). Israelian et al. (2009) confirm former results using the homogeneous and high-quality HARPS GTO sample. They find that about $50 \%$ of 60 solar analogues $\left(T_{\text {eff }}=T_{\odot}^{2} \pm 80 \mathrm{~K}\right)$ without detected planets had $A(\mathrm{Li}) \geq 1.5$, while only 2 out of 24 planet hosts had high Li abundances. Other recent works by Takeda et al. (2010) and Gonzalez et al. (2010) have also reported similar results. Furthermore, Sousa et al. (2010) show that mass and age are not responsible for the observed correlation using the same sample as in Israelian et al. (2009). Doubts about the proposed Li-planet connection have been raised in several works (Ryan 2000; Luck \& Heiter 2006; Baumann et al. 2010; Ghezzi et al. 2010; Ramírez et al. 2012) and argue that the observed behaviour of $\mathrm{Li}$ abundances could be associated to age, mass, or metallicity differences between stars with and without planets. However, this cannot be the ultimate explanation of the observed behaviour of $\mathrm{Li}$ in planet host stars, since several old stellar clusters like M 67 have shown that solar-type stars of very similar age and metallicity present a wide dispersion of Li abundances (Randich et al. 2007; Pasquini et al. 2008; Pace et al. 2012). What is then the principal parameter that accounts for this scatter in $\mathrm{Li}$ abundances?

Lithium is formed in a significant quantity at the primordial nucleosynthesis and is easily destroyed by (p, $\alpha$ )-reactions at 2.5 million $\mathrm{K}$ in the inner layers of solar-type stars. Although Li depletion occurs primarily in the pre-main sequence (PMS), it can also take place in stellar envelopes if any extra mixing process exists. In fact, there is an obvious relation between $T_{\text {eff }}$ and Li-depletion: cooler stars are more Li-depleted because of their thicker convective envelopes (where $\mathrm{Li}$ is brought to hotter layers to be depleted).

It seems that rotation-induced mixing and angular momentum loss are the most efficient processes destroying $\mathrm{Li}$ in solar-type stars on the main sequence (MS; e.g. Zahn 1992; Pinsonneault et al. 1992; Deliyannis \& Pinsonneault 1997). Observations indicate that rapidly rotating stars preserve more $\mathrm{Li}$ than slow rotators of the same mass, as observed in Pleiades (Soderblom et al. 1993; Garcia Lopez et al. 1994) or IC 2602 (Randich et al. 1997). This is also found in solar-type stars by Takeda et al. (2010) and Gonzalez et al. (2010). Other invoked mechanisms are internal waves (e.g. Montalban \& Schatzman 1996) or overshooting mixing, recently claimed as being a primary depletion mechanism (e.g. Xiong \& Deng 2009; Zhang 2012). Some works have also combined several mechanisms showing that the efficiency of rotational mixing is decreased when magnetic fields are taken into account (Eggenberger et al. 2010), as well as when there are internal gravity waves (Charbonnel \& Talon 2005) since they lead to efficient angular momentum redistribution from the core to the envelope.

Other mechanisms directly related to the presence of planets have been proposed as causing additional Li depletion. For instance, Israelian et al. (2004), Chen \& Zhao (2006), and Castro et al. (2009) have suggested that Li depletion could be related to planet migration since it can create a shear instability that produces effective mixing. It is also possible that proto-planetary discs lock a large amount of angular momentum and therefore create some rotational breaking in the host stars during the

\footnotetext{
1 We adopt this term for the sake of simplicity throughout the paper. We warn the reader not to confuse it with the term typically given to non-binary stars.

$2 T_{\odot}=5777 \mathrm{~K}$.
}

PMS inducing an increased mixing (Israelian et al. 2004). This possibility has also been proposed by Bouvier (2008) using simple rotational models: long-lived accretion discs of stars with low initial velocities lead to the formation of a large velocity shear in the base of the convective zone. The velocity gradient in turn triggers hydrodynamical instabilities responsible for enhanced lithium burning on PMS and MS evolution scales. This explanation matches the Li depletion in planet hosts well since we expect long disc lifetimes in order to form planets. The relation of long-lived discs with slow rotation on the ZAMS has also been probed by Eggenberger et al. (2012) as well as the increase of rotational mixing (and thus Li depletion) when differential rotation rises in the stellar interior during PMS. Finally, the infall of planetary material might also affect the mixing processes of those stars by thermohaline convection (Garaud 2011; Théado \& Vauclair 2012), as well as the episodic accretion of planetary material can increase the temperature in the bottom of the convective envelope, hence increase Li depletion (Baraffe \& Chabrier 2010).

The above mentioned theoretical studies and models propose a clear relationship among stellar rotation (PMS or MS), formation and evolution of planets, and surface Li abundances of solar type stars. To discover this relation observationally, homogeneous and precise studies of planet host stars have to be undertaken. In this paper we extend our previous Li work (Israelian et al. 2009) by including new stars observed in HARPS surveys and at other telescopes.

\section{Observations and stellar samples}

The principal sample used in this work is formed by 1111 FGK stars observed within the context of the HARPS GTO programmes to search for planets. The stars in that project were selected from a volume-limited stellar sample observed by the CORALIE spectrograph at La Silla observatory (Udry et al. 2000). The stars were selected to be suitable for radial velocity surveys. They are slow rotating and non-evolved solar type dwarfs with spectral type between F2 and M0 that also do not show a high level of chromospheric activity. The final sample is a combination of three HARPS subsamples hereafter called HARPS-1 (Mayor et al. 2003), HARPS-2 (Lo Curto et al. 2010) and HARPS-4 (Santos et al. 2011). Note that the HARPS-2 planet search programme is the complementation of the previously started CORALIE survey (Udry et al. 2000) to fainter magnitudes and to a larger volume.

The individual spectra of each star were reduced using the HARPS pipeline and then combined with IRAF $^{3}$ after correcting for its radial velocity. The final spectra have a resolution of $R \sim 115000$ and high signal-to-noise ratio (55\% of the spectra have $\mathrm{S} / \mathrm{N}$ higher than 200), depending on the amount and quality of the original spectra. The total sample is composed of 135 stars with planets and 976 stars without detected planets, but in our effective temperature region of interest (5600-5900 K) we have 42 and 235 stars with and without planets. To increase the number of stars with planets we used spectroscopic data for 49 planet hosts which come from different observing runs, listed in Table 1, some of them belonging to the CORALIE survey (see Table 4). The data reduction was made with the IRAF package or with the respective telescopes pipelines. All the images were flat-field-corrected, sky-substracted, and added

IRAF is distributed by National Optical Astronomy Observatories, operated by the Association of Universities for Research in Astronomy, Inc., under contract with the National Science Fundation, USA. 
Table 1. Observing details: telescopes, spectrographs, resolving power, and spectral ranges used in this work.

\begin{tabular}{lccc}
\hline \hline Telescope & Instrument & Resolution & Spectral range \\
& & $\lambda / \delta \lambda$ & $\AA$ \\
\hline 3.6-m ESO La Silla Observatory (Chile) & HARPS & 100000 & $3.800-7.000$ \\
8.2-m Kueyen UT2 (VLT) & UVES & 115000 & $3.000-4.800,4.800-6.800$ \\
2.2-m ESO/MPI telescope & FEROS & 48000 & $3.600-9.200$ \\
3.5-m TNG & SARG & $57000 / 86000$ & $5.100-10.100$ \\
2.6-m Nordic Optical Telescope & FIES & 67000 & $3.700-7.300$ \\
1.93-m OHP & SOPHIE & 75000 & $3.820-6.930$ \\
1.2-m Euler Swiss telescope & CORALIE & 50000 & $3.800-6.800$ \\
4.2-m William Herschel Telescope & UES & 55000 & $4.600-7.800$ \\
\hline
\end{tabular}

Table 2. Definition of the subsamples in this work.

\begin{tabular}{lccccccc}
\hline \hline Name & $\begin{array}{c}T_{\text {eff }} \\
(\mathrm{K})\end{array}$ & {$[\mathrm{Fe} / \mathrm{H}]$} & $\begin{array}{c}\log g \\
\left(\mathrm{~cm} \mathrm{~s}^{-2}\right)\end{array}$ & Planet hosts $^{a}$ & Single stars & $\begin{array}{c}\text { Planet hosts } \\
A(\mathrm{Li})>1.4\end{array}$ & $\begin{array}{c}\text { Single stars } \\
A(\mathrm{Li})>1.4\end{array}$ \\
\hline Solar type & 5600,5900 & $-0.85,0.5$ & $3.8,4.7$ & $43+49$ & $233^{b}$ & $28 \%+5 \%-4 \%$ & $43 \% \pm 3 \%$ \\
Solar analogues & 5697,5857 & $-0.6,0.5$ & $4.24,4.64$ & $18+25$ & 99 & $19 \%+7 \%-4 \%$ & $47 \% \pm 5 \%$ \\
Solar twins & 5697,5857 & $-0.2,0.2$ & $4.24,4.64$ & $9+13$ & 60 & $18 \%+10 \%-5 \%$ & $48 \% \pm 6 \%$ \\
\hline
\end{tabular}

Notes. ${ }^{(a)}$ Planets hosts from HARPS (including the Sun)+planet hosts from other surveys. ${ }^{(b)}$ There are 2 more stars in this $T_{\text {eff }}$ range with metallicities -1.04 and -1.07 shown in Table 6 .

to obtain 1D spectra. Doppler correction was also done. We note that our sample contains $98 \%$ of planets hosts within the range $5600-5900 \mathrm{~K}$ discovered by radial velocities and $77 \%$ if we also consider the transiting planet hosts (Santos et al. 2013).

\section{Analysis}

The stellar atmospheric parameters were taken from Sousa et al. (2008, 2011a,b) for HARPS stars and from Santos et al. (2004, 2005), Sousa et al. (2006) and Mortier et al. (2013) for the rest of the planet hosts. The errors of the parameters from Santos et al. $(2004,2005)$ are of the order of $44 \mathrm{~K}$ for $T_{\text {eff }}, 0.11$ dex for $\log g, 0.08 \mathrm{~km} \mathrm{~s}^{-1}$ for $\xi_{\mathrm{t}}, 0.06$ dex for metallicity and $0.05 M_{\odot}$ for the masses. From Sousa et al. (2008, 2011a,b), the typical errors are $30 \mathrm{~K}$ for $T_{\text {eff }}, 0.06$ dex for $\log g, 0.08 \mathrm{~km} \mathrm{~s}^{-1}$ for $\xi_{\mathrm{t}}$, and 0.03 dex for metallicity. We refer to those works for further details in the parameters determination and errors. All the sets of parameters were determined in a consistent way so as to reduce at maximum the systematic errors. We note here the uniformity of the adopted stellar parameters as discussed in Sect. 5 of Sousa et al. (2008). Our group is continuously updating the stellar parameters of stars under consideration (Sousa et al. 2008, 2011a,b) and has created an online catalogue (Santos et al. 2013) to guarantee a homogeneous and precise study of stellar abundances. This is especially important for Li when we have clearly limited the study to solar analogues.

Li abundances, $A(\mathrm{Li})^{4}$, were derived by standard LTE analysis using spectral synthesis with the revised version of the spectral synthesis code MOOG2010 (Sneden 1973) and a grid of Kurucz ATLAS9 atmospheres with overshooting (Kurucz 1993). We used the linelist from Ghezzi et al. (2009) though we applied a slight correction to the $\log g f$ (to -2.278) of FeI line at $6707.4 \AA$ to adjust the Kurucz Solar Flux Atlas spectrum (Kurucz et al. 1984). We neglected possible ${ }^{6} \mathrm{Li}$ contributions. We did not apply NLTE corrections since for this kind of star they are quantitatively insignificant compared to the large dispersion of $\mathrm{Li}$ abundances or to a conservative typical error of 0.1 dex (Takeda \& Kawanomoto 2005; Ramírez et al. 2012).

\footnotetext{
$\overline{4 A(\mathrm{Li})=\log [N(\mathrm{Li}) / N(\mathrm{H})]}+12$.
}

Some examples of spectral synthesis are shown in Fig. 1. All abundances and their errors are listed in Tables 3, 4, and $6^{5}$.

Stellar masses and ages were derived by using the stellar evolutionary models from the Padova group computed with the web interface $^{6}$ dealing with stellar isochrones and their derivatives to the stars of our sample. The values for $T_{\text {eff }}$ and $[\mathrm{Fe} / \mathrm{H}]$ were taken from the previously mentioned works and $V$ magnitudes and parallaxes come from the Hipparcos database. For those stars with no values in Hipparcos database we used other sources as Simbad database ${ }^{7}$ or The Extrasolar Planets Encyclopaedia ${ }^{8}$ (Schneider et al. 2011).

\section{Discussion}

In Fig. 2 we present a general overview of the behaviour of $\mathrm{Li}$ as a function of effective temperature for solar type stars. We can see in the plot the ranges in $T_{\mathrm{eff}},[\mathrm{Fe} / \mathrm{H}]$ and gravity for the stars in this sample (see also Table 2). As expected, below $5650 \mathrm{~K}$, most of the stars have severe depleted $\mathrm{Li}$ abundances regardless of whether they have planets or not. These stars have deeper convective envelopes that allow the material to reach the Li-burning layers on the MS. However, a similar depletion of $\mathrm{Li}$ is also observed in stars with slightly higher temperatures (around solar $T_{\text {eff }}$ ). Standard models (Deliyannis et al. 1990; Pinsonneault 1997), which only consider mixing by convection, do not predict the Li depletion observed in solar-type stars. Indeed, the location of the base of the solar convection zone inferred from helioseismology is not hot enough to burn Li on the MS (ChristensenDalsgaard et al. 1991).

On the other hand, when the temperature is close to $5900 \mathrm{~K}$, most of the stars have preserved higher amounts of Li (due to their shallower convective envelopes) but still present signs of depletion that are not expected from canonical models either (e.g. Pinsonneault 1997). Therefore, there must be other

\footnotetext{
5 We note that there are some stars in common between the three used HARPS samples. We only used the best available spectrum for each of those, with its corresponding parameters as shown in these tables.

6 http://stev.oapd.inaf.it/cgi-bin/param

7 http://simbad.u-strasbg.fr/simbad/sim-fid

8 http://exoplanet.eu/catalog/
} 
Table 3. Li abundances for stars with planets from HARPS GTO samples.

\begin{tabular}{|c|c|c|c|c|c|c|c|c|c|}
\hline Star & $\begin{array}{l}T_{\text {eff }} \\
(\mathrm{K}) \\
\end{array}$ & $\begin{array}{c}\log g \\
\left(\mathrm{~cm} \mathrm{~s}^{-2}\right)\end{array}$ & $\begin{array}{c}\xi_{\mathrm{t}} \\
\left(\mathrm{km} \mathrm{s}^{-1}\right)\end{array}$ & {$[\mathrm{Fe} / \mathrm{H}]$} & $\begin{array}{r}\text { Age } \\
(\mathrm{Gyr})\end{array}$ & $\begin{array}{l}\text { Mass } \\
\left(M_{\odot}\right)\end{array}$ & $A(\mathrm{Li})$ & Error & $\begin{array}{c}M \sin i \\
\left(M_{J}\right)\end{array}$ \\
\hline \multicolumn{10}{|l|}{ HARPS-1 } \\
\hline HD $1461^{a}$ & 5765 & 4.38 & 0.97 & 0.19 & 3.73 & 1.05 & 0.68 & 0.10 & 0.024 \\
\hline HD $4308^{a}$ & 5644 & 4.38 & 0.90 & -0.34 & 11.72 & 0.82 & 0.96 & 0.06 & 0.041 \\
\hline HD 16141 & 5806 & 4.19 & 1.11 & 0.16 & 6.55 & 1.07 & 1.25 & 0.10 & 0.215 \\
\hline HD $16417^{a}$ & 5841 & 4.16 & 1.18 & 0.13 & 5.99 & 1.12 & 1.80 & 0.04 & 0.069 \\
\hline HD 20782 & 5774 & 4.37 & 1.00 & -0.06 & 9.21 & 0.94 & $<0.47$ & - & 1.900 \\
\hline HD 28185 & 5667 & 4.42 & 0.94 & 0.21 & 3.38 & 1.02 & $<0.26$ & - & 5.700 \\
\hline HD $31527^{a}$ & 5898 & 4.45 & 1.09 & -0.17 & 7.57 & 0.95 & 1.91 & 0.03 & 0.052 \\
\hline HD $38858^{a}$ & 5733 & 4.51 & 0.94 & -0.22 & 8.48 & 0.89 & 1.49 & 0.05 & 0.096 \\
\hline HD $45184^{a}$ & 5869 & 4.47 & 1.03 & 0.04 & 2.28 & 1.05 & 2.06 & 0.04 & 0.040 \\
\hline HD 47186 & 5675 & 4.36 & 0.93 & 0.23 & 4.57 & 1.02 & 0.58 & 0.10 & 0.351 \\
\hline HD 65216 & 5612 & 4.44 & 0.78 & -0.17 & 6.20 & 0.89 & 1.23 & 0.05 & 1.210 \\
\hline HD 66428 & 5705 & 4.31 & 0.96 & 0.25 & 5.26 & 1.03 & $<0.71$ & - & 2.820 \\
\hline HD 70642 & 5668 & 4.40 & 0.82 & 0.18 & 2.69 & 1.02 & $<0.46$ & - & 2.000 \\
\hline HD 92788 & 5744 & 4.39 & 0.95 & 0.27 & 1.81 & 1.05 & 0.62 & 0.07 & 3.860 \\
\hline HD $96700^{a}$ & 5845 & 4.39 & 1.04 & -0.18 & 10.53 & 0.93 & 1.27 & 0.08 & 0.040 \\
\hline HD 102117 & 5657 & 4.31 & 0.99 & 0.28 & 8.40 & 1.01 & 0.52 & 0.10 & 0.172 \\
\hline HD $102365^{a}$ & 5629 & 4.44 & 0.91 & -0.29 & 11.32 & 0.85 & $<0.30$ & - & 0.050 \\
\hline HD 107148 & 5805 & 4.40 & 0.93 & 0.31 & 3.63 & 1.07 & $<1.34$ & - & 0.210 \\
\hline HD 114729 & 5844 & 4.19 & 1.23 & -0.28 & 11.00 & 0.95 & 1.95 & 0.04 & 0.840 \\
\hline HD 117207 & 5667 & 4.32 & 1.01 & 0.22 & 6.31 & 1.01 & $<0.12$ & - & 2.060 \\
\hline HD 134987 & 5740 & 4.30 & 1.08 & 0.25 & 6.29 & 1.04 & $<0.60$ & - & 1.590 \\
\hline HD 141937 & 5893 & 4.45 & 1.00 & 0.13 & 1.08 & 1.09 & 2.35 & 0.04 & 9.700 \\
\hline HD 147513 & 5858 & 4.50 & 1.03 & 0.03 & 0.64 & 1.05 & 2.05 & 0.05 & 1.210 \\
\hline HD 160691 & 5780 & 4.27 & 1.09 & 0.30 & 6.36 & 1.07 & 0.98 & 0.10 & 1.814 \\
\hline HD 190647 & 5639 & 4.18 & 0.99 & 0.23 & 8.47 & 1.04 & $<0.51$ & - & 1.900 \\
\hline HD 202206 & 5757 & 4.47 & 1.01 & 0.29 & 1.97 & 1.06 & 1.37 & 0.05 & 17.400 \\
\hline HD 204313 & 5776 & 4.38 & 1.00 & 0.18 & 3.03 & 1.05 & $<0.52$ & - & 3.550 \\
\hline HD 222582 & 5779 & 4.37 & 1.00 & -0.01 & 7.48 & 0.97 & 0.85 & 0.15 & 7.750 \\
\hline HD 126525 & 5638 & 4.37 & 0.90 & -0.10 & 9.60 & 0.89 & $<-0.01$ & - & 0.224 \\
\hline HD 134606 & 5633 & 4.38 & 1.00 & 0.27 & 7.09 & 0.99 & $<0.39$ & - & 0.121 \\
\hline HD $136352^{a}$ & 5664 & 4.39 & 0.90 & -0.34 & 11.73 & 0.84 & $<-0.10$ & - & 0.036 \\
\hline HD 150433 & 5665 & 4.43 & 0.88 & -0.36 & 11.43 & 0.82 & $<0.27$ & - & 0.168 \\
\hline HD $189567^{a}$ & 5726 & 4.41 & 0.95 & -0.24 & 11.55 & 0.87 & $<0.18$ & - & 0.032 \\
\hline HD 215456 & 5789 & 4.10 & 1.19 & -0.09 & 8.37 & 1.05 & 2.31 & 0.04 & 0.246 \\
\hline \multicolumn{10}{|l|}{ HARPS-4 } \\
\hline HD 171028 & 5671 & 3.84 & 1.24 & -0.48 & - & - & $<0.04$ & - & 1.980 \\
\hline HD 181720 & 5792 & 4.25 & 1.16 & -0.53 & 11.22 & 0.92 & 1.93 & 0.02 & 0.370 \\
\hline \multicolumn{10}{|l|}{ HARPS-2 } \\
\hline HD 6718 & 5723 & 4.44 & 0.84 & -0.07 & 6.54 & 0.95 & $<0.47$ & - & 1.560 \\
\hline HD 28254 & 5653 & 4.15 & 1.08 & 0.36 & 7.75 & 1.06 & $<0.59$ & - & 1.160 \\
\hline HD 30177 & 5601 & 4.34 & 0.89 & 0.37 & 5.66 & 0.99 & $<0.51$ & - & 7.700 \\
\hline HD 44219 & 5766 & 4.20 & 1.06 & 0.04 & 8.43 & 1.01 & 1.23 & 0.10 & 0.580 \\
\hline HD $109271^{a}$ & 5783 & 4.28 & 0.97 & 0.10 & 7.30 & 1.05 & 1.42 & 0.08 & 0.076 \\
\hline HD 207832 & 5718 & 4.45 & 0.86 & 0.15 & 1.90 & 1.01 & $<1.04$ & - & 0.730 \\
\hline
\end{tabular}

Notes. Parameters from Sousa et al. (2008, 2011a,b). The minimum mass of the most massive planet in the system is indicated in the last column (taken from The Extrasolar Planets Encyclopaedia, Schneider et al. 2011). ${ }^{(a)}$ Stars which only host Neptune and Earth-like planets.

mechanisms such as rotationally induced mixing (Pinsonneault et al. 1990), diffusion (Richer \& Michaud 1993), internal waves (Montalbán \& Schatzman 2000), overshooting (e.g. Xiong \& Deng 2009) or the combination of several (e.g. Chaboyer et al. 1995; Charbonnel \& Talon 2005), that account for the destruction of Li observed in solar-type stars in open clusters and in the field (e.g. Pace et al. 2012).

As seen in Fig. 2, there is a high dispersion in $\mathrm{Li}$ abundances in the $T_{\text {eff }}$ interval $5600-5900 \mathrm{~K}$. Most of the planet hosts have destroyed their $\mathrm{Li}$ in this $T_{\text {eff }}$ window. To better appreciate the effects of planets on Li depletion we now focus in the solar temperature range, $T_{\text {eff }}=T_{\odot} \pm 80 \mathrm{~K}$, where previous works found differences between both groups of stars (e.g. Israelian et al. 2009). In this range of temperatures the convective envelope is not as deep as in cooler stars but lies very close to the Li burning layer. If some mechanism exists that is capable of producing an extra mixing (even if it is not very intense), $\mathrm{Li}$ will therefore suffer some depletion. As a consequence, this type of stars is very sensitive to non-standard mixing processes.

Below we discuss the dependence of Li depletion on several parameters. It is important to note that to make a meaningful comparison of planet hosts and single stars, we have to deal with solar-type stars in the MS. Therefore, we removed from our sample of planet hosts some stars showing activity and/or with very young ages such as HD 81040, which has a disc (Sozzetti et al. 2006); HD 70573 (Setiawan et al. 2007); or 
E. Delgado Mena et al.: Li depletion in solar analogues with exoplanets

Table 4. Planet hosts stars not belonging to the HARPS-GTO sample.

\begin{tabular}{|c|c|c|c|c|c|c|c|c|c|c|c|}
\hline Star & $\begin{array}{l}T_{\text {eff }} \\
(\mathrm{K})\end{array}$ & $\begin{array}{c}\log g \\
\left(\mathrm{~cm} \mathrm{~s}^{-2}\right) \\
\end{array}$ & $\begin{array}{c}\xi_{\mathrm{t}} \\
\left(\mathrm{km} \mathrm{s}^{-1}\right)\end{array}$ & {$[\mathrm{Fe} / \mathrm{H}]$} & $\begin{array}{r}\text { Age } \\
(\mathrm{Gyr})\end{array}$ & $\begin{array}{l}\text { Mass } \\
\left(M_{\odot}\right)\end{array}$ & $A(\mathrm{Li})$ & Error & $\begin{array}{c}M \sin i \\
\left(M_{J}\right)\end{array}$ & Flag & Reference \\
\hline HD 45350 & 5650 & 4.29 & 1.08 & 0.28 & 8.22 & 1.01 & $<0.52$ & - & 1.790 & [6] & this work \\
\hline HD 79498 & 5814 & 4.40 & 1.09 & 0.24 & 1.10 & 1.08 & 1.28 & 0.10 & 1.340 & [6] & this work \\
\hline HD $179079^{a}$ & 5742 & 4.11 & 1.22 & 0.26 & 6.86 & 1.09 & 2.01 & 0.05 & 0.080 & [6] & this work \\
\hline HIP 14810 & 5601 & 4.43 & 0.96 & 0.26 & 4.35 & 0.99 & $<0.84$ & - & 3.880 & [1] & this work \\
\hline HD 4113 & 5688 & 4.40 & 1.08 & 0.20 & 5.39 & 1.01 & $<0.86$ & - & 1.560 & {$[5]$} & Tamuz et al. (2008) \\
\hline HD 4203 & 5636 & 4.23 & 1.12 & 0.40 & 8.23 & 1.01 & $<0.65$ & - & 2.070 & [3] & Santos et al. (2004) \\
\hline HD 6434 & 5835 & 4.60 & 1.53 & -0.52 & 10.21 & 0.86 & $<0.79$ & - & 0.390 & [3] & Santos et al. (2004) \\
\hline HD 12661 & 5702 & 4.33 & 1.05 & 0.36 & 5.84 & 1.02 & $<0.63$ & - & 2.300 & [8] & Santos et al. (2004) \\
\hline HD 49674 & 5644 & 4.37 & 0.89 & 0.33 & 2.13 & 1.01 & $<1.00$ & - & 0.115 & [4] & Santos et al. (2004) \\
\hline HD 73526 & 5699 & 4.27 & 1.26 & 0.27 & 7.28 & 1.05 & 0.63 & 0.10 & 2.900 & [1] & Santos et al. (2004) \\
\hline HD 76700 & 5737 & 4.25 & 1.18 & 0.41 & 6.80 & 1.05 & 1.27 & 0.10 & 0.190 & [1] & Santos et al. (2004) \\
\hline HD 106252 & 5899 & 4.34 & 1.08 & -0.01 & 3.52 & 1.03 & 1.71 & 0.05 & 7.560 & [1] & Santos et al. (2004) \\
\hline HD 109749 & 5899 & 4.31 & 1.13 & 0.32 & 2.98 & 1.12 & 2.24 & 0.05 & 0.280 & [5] & Sousa et al. (2006) \\
\hline HD 114762 & 5884 & 4.22 & 1.31 & -0.70 & 11.61 & 0.87 & 2.07 & 0.03 & 10.980 & [1] & Santos et al. (2004) \\
\hline HD 154857 & 5610 & 4.02 & 1.30 & -0.23 & 4.52 & 1.22 & 1.68 & 0.03 & 1.800 & [3] & Santos et al. (2005) \\
\hline HD 168443 & 5617 & 4.22 & 1.21 & 0.06 & 9.68 & 1.01 & $<0.45$ & - & 17.193 & [4] & Santos et al. (2004) \\
\hline HD 178911B & 5600 & 4.44 & 0.95 & 0.27 & 5.38 & 0.99 & $<0.94$ & - & 6.292 & [4] & Santos et al. (2004) \\
\hline HD 186427 & 5772 & 4.40 & 1.07 & 0.08 & 6.23 & 1.00 & $<0.52$ & - & 1.680 & [4] & Santos et al. (2004) \\
\hline HD 187123 & 5845 & 4.42 & 1.10 & 0.13 & 4.53 & 1.05 & $<0.54$ & - & 1.990 & [4] & Santos et al. (2004) \\
\hline HD 188015 & 5793 & 4.49 & 1.14 & 0.30 & 2.22 & 1.07 & $<0.87$ & - & 1.260 & [3] & Santos et al. (2005) \\
\hline HD 195019 & 5859 & 4.32 & 1.27 & 0.09 & 6.53 & 1.07 & 1.53 & 0.06 & 3.700 & [8] & Santos et al. (2004) \\
\hline HD 216437 & 5887 & 4.30 & 1.31 & 0.25 & 4.65 & 1.15 & 1.99 & 0.06 & 1.820 & [1] & Santos et al. (2004) \\
\hline HD 217014 & 5804 & 4.42 & 1.20 & 0.20 & 3.94 & 1.06 & 1.29 & 0.10 & 0.468 & [3] & Santos et al. (2004) \\
\hline HD 217107 & 5645 & 4.31 & 1.06 & 0.37 & 7.30 & 0.99 & $<0.61$ & - & 2.490 & [3] & Santos et al. (2004) \\
\hline HD 9446 & 5793 & 4.53 & 1.01 & 0.09 & 2.49 & 1.03 & 1.82 & 0.05 & 1.820 & [7] & Hébrard et al. (2010) \\
\hline HD 109246 & 5844 & 4.46 & 1.01 & 0.10 & 2.08 & 1.04 & 1.58 & 0.10 & 0.770 & [7] & Boisse et al. (2010) \\
\hline Kepler-17 & 5781 & 4.53 & 1.73 & 0.26 & 3.68 & 1.07 & $<1.38$ & - & 2.450 & [7] & Bonomo et al. (2012) \\
\hline KOI-204 & 5757 & 4.15 & 1.75 & 0.26 & 4.03 & 1.06 & $<1.38$ & - & 1.020 & [7] & Bonomo et al. (2012) \\
\hline $\mathrm{XO}-1$ & 5754 & 4.61 & 1.07 & -0.01 & - & - & 1.33 & 0.12 & 0.900 & [4] & Ammler-von Eiff et al. (2009) \\
\hline HD 23127 & 5891 & 4.23 & 1.26 & 0.41 & 4.32 & 1.20 & 2.67 & 0.02 & 1.500 & [1] & Santos et al. (2013) \\
\hline HD 24040 & 5840 & 4.30 & 1.14 & 0.20 & 5.36 & 1.09 & 1.17 & 0.10 & 4.010 & [1] & Santos et al. (2013) \\
\hline HD 27631 & 5700 & 4.37 & 1.00 & -0.11 & 5.99 & 0.93 & $<-0.07$ & - & 1.450 & [3] & Santos et al. (2013) \\
\hline HD 96167 & 5823 & 4.16 & 1.28 & 0.38 & 4.47 & 1.22 & 1.59 & 0.08 & 0.680 & [3] & Santos et al. (2013) \\
\hline HD 98649 & 5714 & 4.37 & 1.01 & -0.03 & 4.24 & 0.96 & $<-0.54$ & - & 6.800 & [3] & Santos et al. (2013) \\
\hline HD 126614 & 5601 & 4.25 & 1.17 & 0.50 & 8.57 & 0.99 & $<0.57$ & - & 0.380 & [1] & Santos et al. (2013) \\
\hline HD 129445 & 5646 & 4.28 & 1.14 & 0.37 & 4.95 & 1.00 & $<0.77$ & - & 1.600 & [3] & Santos et al. (2013) \\
\hline HD 152079 & 5785 & 4.38 & 1.09 & 0.29 & 2.98 & 1.06 & $<1.00$ & - & 3.000 & [2] & Santos et al. (2013) \\
\hline HD 154672 & 5743 & 4.27 & 1.08 & 0.25 & 6.94 & 1.06 & $<0.61$ & - & 5.020 & [1] & Santos et al. (2013) \\
\hline HD 170469 & 5845 & 4.28 & 1.17 & 0.30 & 4.54 & 1.09 & 1.23 & 0.10 & 0.670 & [1] & Santos et al. (2013) \\
\hline CoRoT-9 & 5613 & 4.35 & 0.90 & -0.02 & 5.15 & 0.94 & $<0.88$ & - & 0.840 & [2] & Mortier et al. (2013) \\
\hline CoRoT-12 & 5715 & 4.66 & 1.07 & 0.17 & 4.31 & 1.01 & $<1.65$ & - & 0.917 & [2] & Mortier et al. (2013) \\
\hline WASP-5 & 5785 & 4.54 & 0.96 & 0.17 & 3.34 & 1.04 & $<1.23$ & - & 1.637 & {$[1]$} & Mortier et al. (2013) \\
\hline WASP-8 & 5690 & 4.42 & 1.25 & 0.29 & 3.22 & 1.03 & 1.54 & 0.15 & 2.244 & [3] & Mortier et al. (2013) \\
\hline WASP-16 & 5726 & 4.34 & 0.97 & 0.13 & 4.43 & 1.02 & $<0.62$ & - & 0.855 & [2] & Mortier et al. (2013) \\
\hline WASP-25 & 5736 & 4.52 & 1.11 & 0.06 & 3.94 & 1.00 & 1.69 & 0.05 & 0.580 & [2] & Mortier et al. (2013) \\
\hline WASP-34 & 5704 & 4.35 & 0.97 & 0.08 & 5.15 & 0.99 & $<0.15$ & - & 0.590 & [3] & Mortier et al. (2013) \\
\hline WASP-56 & 5797 & 4.44 & 1.19 & 0.43 & 3.01 & 1.07 & 1.65 & 0.10 & 0.600 & [3] & Mortier et al. (2013) \\
\hline WASP-63 & 5715 & 4.29 & 1.28 & 0.28 & 4.36 & 1.04 & 1.45 & 0.07 & 0.380 & [3] & Mortier et al. (2013) \\
\hline WASP-77A & 5605 & 4.37 & 1.09 & 0.07 & 5.55 & 0.95 & $<0.59$ & - & 1.760 & [3] & Mortier et al. (2013) \\
\hline
\end{tabular}

Notes. The minimum mass of the most massive planet in each system is taken from The Extrasolar Planets Encyclopaedia, Schneider et al. (2011). Flag: [1] UVES; [2] HARPS; [3] FEROS; [4] SARG; [5] CORALIE; [6] NOT; [7] SOPHIE; [8] UES. ${ }^{(a)}$ Stars which only host Neptune and Earth-like planets.

Corot-2 (Alonso et al. 2008), as well as subgiants or slightly evolved stars such as HD 118203 and HD 149143 (da Silva et al. 2006), HD 38529 (Fischer et al. 2001), HD 48265 (Minniti et al. 2009), HD 175167 (Arriagada et al. 2010), and HD 219828 (Melo et al. 2007). Some of these stars have been included by other authors (Gonzalez et al. 2010; Ghezzi et al. 2010; Ramírez et al. 2012) in previous works about Li depletion and planets; however, we prefer to discard them since they are in different evolutionary stages. For instance, a subgiant with solar $T_{\text {eff }}$ had a higher temperature when it was on the MS and thus different Li content.

We want to stress that our comparison sample of single stars is formed by stars that have been surveyed over years (with some of the most precise planet search projects) and no planets have been detected so far. We are confident that most of those stars do not host nearby giant planets. We cannot rule out that most of single stars in our sample host low-mass planets that are below the detection capabilities of HARPS. It is also possible that 


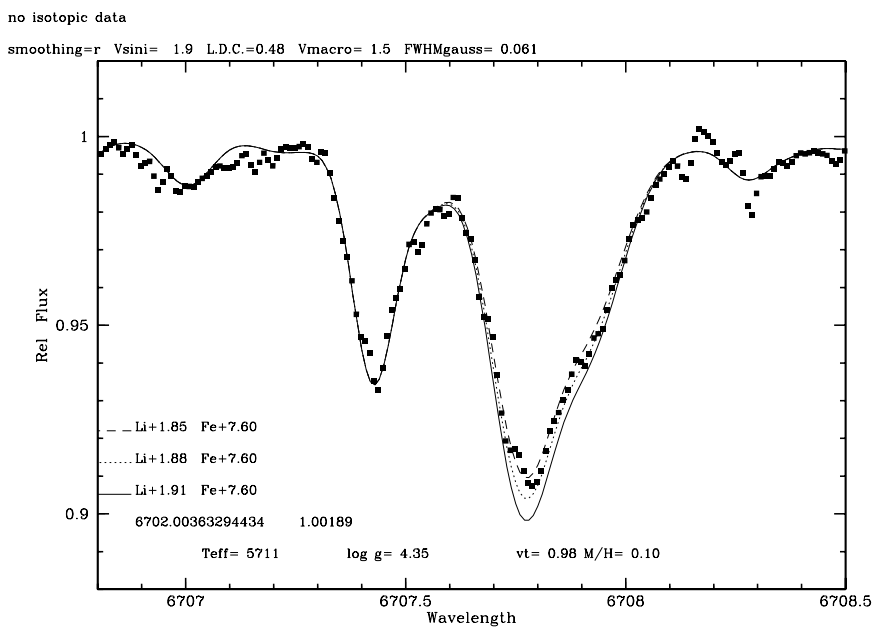

no isotopic data

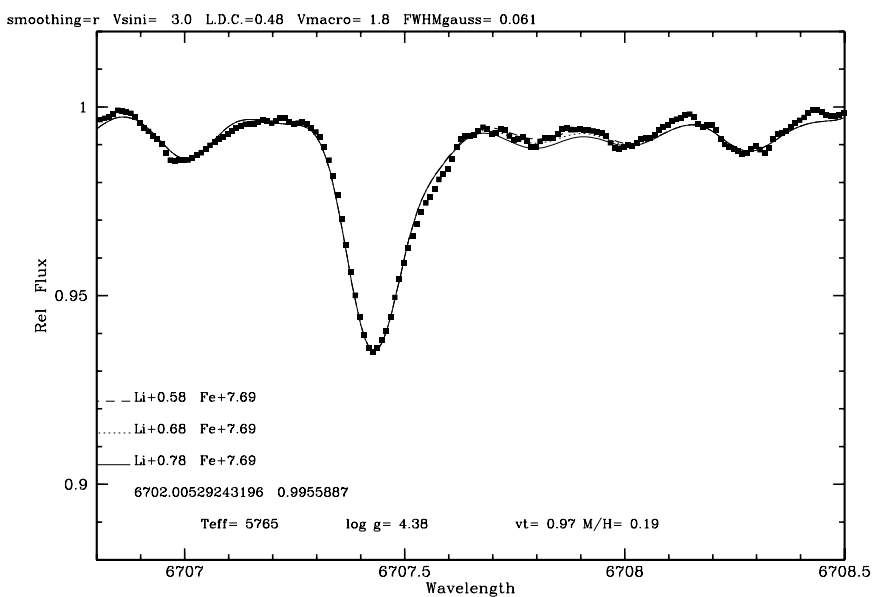

no isotopic data

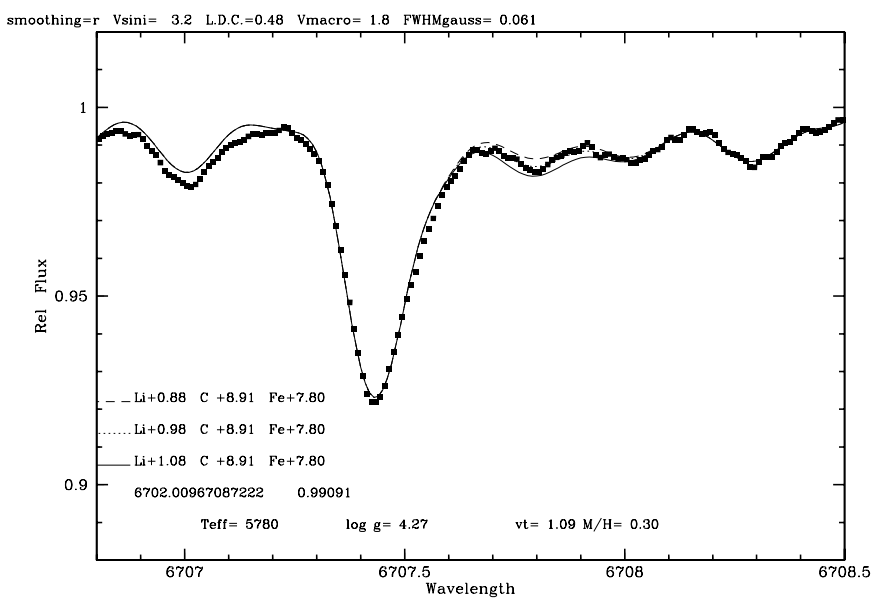

Fig. 1. Spectral synthesis around the Li region for the stars HD 96423 , HD 1461, and HD 160691.

some of these stars have long-period giant planets yet to be discovered. This is an important point, since some earlier studies (Ryan 2000; Chen \& Zhao 2006; Luck \& Heiter 2006; Gonzalez et al. 2010; Baumann et al. 2010; Ramírez et al. 2012) were using field stars as single stars not included in planet-search surveys, but they could have giant planets and thus are not the best targets to be used as real comparison stars. We also point that by keeping the comparison sample from HARPS and adding more planet hosts from other surveys, our results will be statistically

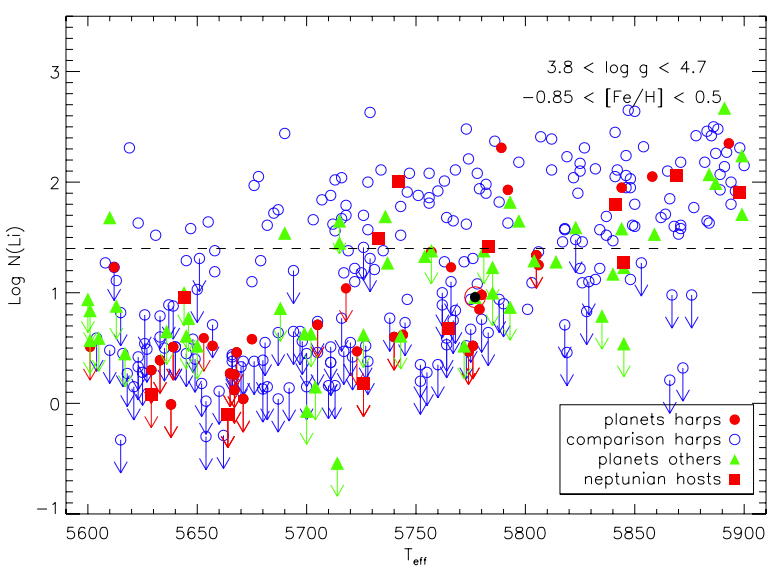

Fig. 2. Lithium abundances vs. $T_{\text {eff }}$ for planet host stars (red filled circles) and single stars (blue open circles) from HARPS, together with other planet hosts (green triangles). Squares are stars that only host Neptunian- or Super-Earth-type planets. Down arrows represent $A(\mathrm{Li})$ upper limits. The straight line at $A(\mathrm{Li})=1.4$ matches the upper envelope of the lower limits for most of our stars.

more significant. Nevertheless, we differentiate those stars in the plots to show that we still keep the homogeneity of the study.

\subsection{Li and log g: cleaning the sample from evolved stars}

Our sample is mainly composed of $\mathrm{G}$ dwarfs with $\log g$ values between 4.1 and 4.6 (Fig. 3). There are several stars with $\log g$ values between 4.1 and 4.2 with high $\mathrm{Li}$ abundances, opposite to what is expected by the general trend starting at 4.2 dex. This has already been noticed in Baumann et al. (2010). Indeed, all the giant planet hosts with high Li have low $\log g$ values except HD $9446\left(T_{\text {eff }}=5793 \mathrm{~K}, A(\mathrm{Li})=1.82\right)$, and HD $181720\left(T_{\text {eff }}=5792 \mathrm{~K}, A(\mathrm{Li})=1.93\right)$. This last planet host has $\log g=4.25$ but $L=2.65 L_{\odot}$ and an age of $11.2 \mathrm{Gyr}$, thus it is possible that this star is entering the subgiant phase. The general trend in Fig. 3 shows a decrease in Li abundances with decreasing $\log g$ in the range $4.2<\log g<4.7$, since these stars with lower $\log g$ are older and have had more time to deplete their Li. If we split this plot into different metallicity bins we still observe this effect.

To check the evolutionary status of those stars we plot them in an HR diagram, together with some evolutionary tracks from Bertelli et al. (2008) and Girardi et al. (2000). The luminosity was computed by considering the HIPPARCos parallaxes, $V$ magnitude, and the bolometric correction as in Sousa et al. (2008). In Fig. 4 we can see that those stars with lower $\log g$ values have much higher luminosities on average and thus may be slightly evolved from the MS. The higher Li abundances of these stars could be explained by the fact that they were hotter when they were on the MS and so did not deplete so much Li. Moreover, there are claims (Deliyannis et al. 1990) that such early evolution may bring up Li to the photosphere in subgiant stars from a reservoir (hidden below the base of the convective envelope) that could have been formed due to microscopic diffusion during the lifetime in the MS. Therefore, to avoid these possibly evolved stars, we restrict our study to stars with higher $\log g$ values, and more specifically to stars with $\log g=\log g_{\odot}{ }^{9} \pm 0.2$, as showed in Fig. 3. We note that $81 \%$ of our stars with $T_{\text {eff }}=$ $T_{\odot} \pm 80 \mathrm{~K}$ also have $\log g=\log g_{\odot} \pm 0.2$. We will call this subsample "solar analogues" (see Table 2).

${ }^{9} \log g_{\odot}=4.44 \mathrm{dex}$ 


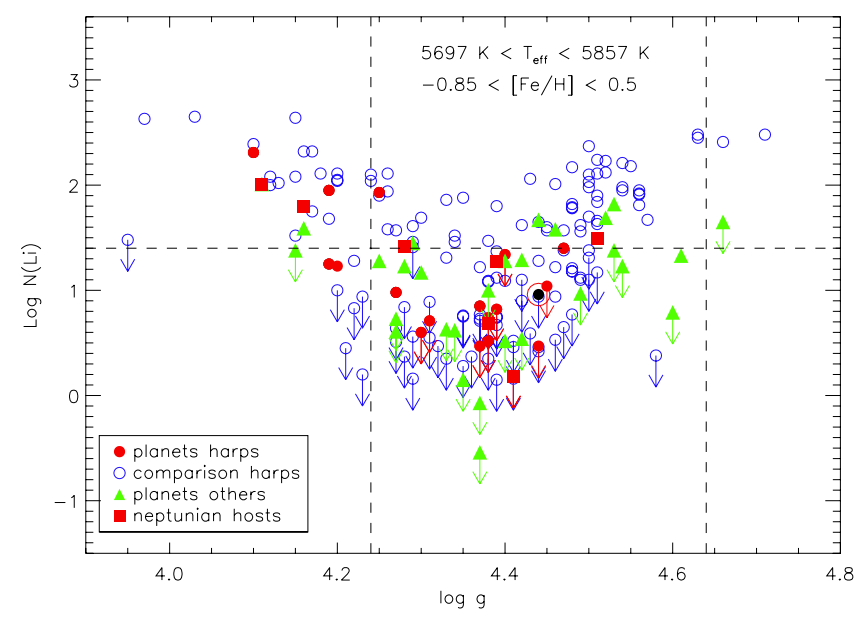

Fig. 3. Lithium abundances vs. $\log g$ for solar type stars. Symbols as in Fig. 2. The vertical lines denote the range in $\log g$ for our solar analogues and solar twins.

\section{2. $\mathrm{Li}$ and $T_{\mathrm{eff}}$}

In Fig. 5 we plot the final sample of solar analogues (see Table 2). We have made a cutoff in $[\mathrm{Fe} / \mathrm{H}]=-0.6$ because our most metal-poor planet host has a metallicity of -0.53 . In any case, only $10 \%$ of our single stars within solar $T_{\text {eff }}$ and $\log g$ range have $[\mathrm{Fe} / \mathrm{H}]<-0.6$. This sample of solar analogues is composed of 43 planet hosts (including the Sun), from which only eight (i.e. $<19 \%$ ) have $\mathrm{Li}$ abundance higher than 1.4. These stars have ages from 2 to $11 \mathrm{Gyr}$ and metallicities from -0.53 to 0.43 . All of them except HD 9446 have planets with masses lower than Jupiter's (see Sect. 4.7).

On the other hand, there are 99 stars without known giant planets, from which $47 \%$ have $\mathrm{Li}$ abundances higher than 1.4 . Therefore, for this $T_{\text {eff }}$ range, there is a clear lack of planet hosts with high $\mathrm{Li}$ content. This fact is not caused by a difference in $T_{\mathrm{eff}}$, so the dependence on other parameters will be analysed later. We stress that given the homogeneous nature of the HARPS sample (and CORALIE survey) there is no reason a priori to expect that planet hosts should have lower $\mathrm{Li}$ in this domain (see Sect. 4.5). "Single" stars are homogeneously distributed in this plot but planet hosts are not, although all of them have similar temperatures, gravities, and metallicities and are all on the MS.

\section{3. $\mathrm{Li}$ and $[\mathrm{Fe} / \mathrm{H}]$}

In Fig. 6 we show $\mathrm{Li}$ abundances as a function of metallicity for what we defined above as solar analogues. Most of the planet hosts present high $[\mathrm{Fe} / \mathrm{H}]$ values, although a few of them reach values lower than -0.5 . The increase in metal opacities in solar-type stars is responsible for the transition between radiative and convective energy transport. Thus, stars with more metals (at a given mass) are expected to have deeper convective envelopes that favour Li depletion, though this assumption might not be correct (Pinsonneault et al. 2001). Therefore, we could first think that the only reason for planet hosts to be Li depleted is that they are metal rich. This might be true for very metal rich stars. We can see in Fig. 6 how above 0.2 dex both stars with and without planets deplete a lot of lithium though we still can detect $\mathrm{Li}$ absorption in some stars. This fact suggests that in our solar $T_{\text {eff }}$ range, high metallicity is also playing a role in lithium depletion, which could be comparable to the role

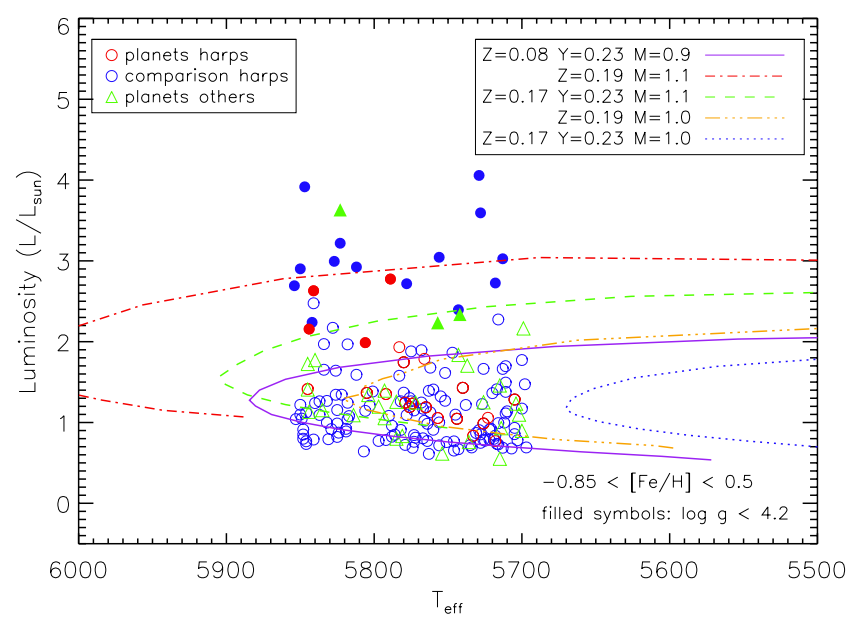

Fig. 4. HR diagram for solar-type stars. Evolutionary tracks are from Girardi et al. (2000); Bertelli et al. (2008). Filled symbols are stars with $\log g$ values lower than 4.2.

of planets, so we cannot extract any conclusion from the most metallic stars. However, we can see in the plot that at lower metallicities most of the planet hosts also present low Li abundances, while single stars are homogeneously spread along high and low $\mathrm{Li}$ abundances. Indeed, if we focus on solar twins (metallicities within 0.2 dex of the solar value, see Table 2 ) only 4 out of 22 planet hosts show Li higher than 1.4, while 29 out of 60 single stars have $A(\mathrm{Li})>1.4$. We can also observe that the $[\mathrm{Fe} / \mathrm{H}]$ distribution at low and high $\mathrm{Li}$ abundances of both groups is quite homogeneous. We do not find any trend of $\mathrm{Li}$ with metallicity in this region $(-0.2<[\mathrm{Fe} / \mathrm{H}]<0.2)$. Therefore, we can be sure that for solar twins $[\mathrm{Fe} / \mathrm{H}]$ is not playing a major role in the depletion of $\mathrm{Li}$.

\subsection{Li and stellar mass}

On the main sequence, stellar mass is directly correlated with $T_{\text {eff }}$ (with a dependence on $[\mathrm{Fe} / \mathrm{H}]$ ), i.e. mass increases with $T_{\text {eff }}$, although solar type stars show a wide spread in masses. However, it is not correct to use only mass as a parameter to constrain solar analogues because some stars with masses and $[\mathrm{Fe} / \mathrm{H}]$ close to solar have high temperatures (up to $6000 \mathrm{~K}$ ) hence fall out of our solar range. We prefer to limit our sample by their $T_{\text {eff }}$ since it is a parameter directly observed and its determination has lower uncertainties than the determination of mass, which in turn depends on the uncertainties of the stellar parameters and metallicity and lies on the theoretical evolutionary tracks.

We have seen in the previous section that to discard a metallicity effect on $\mathrm{Li}$ abundances we have to exclude metal rich stars $([\mathrm{Fe} / \mathrm{H}]>0.2)$ from our sample. On the other hand, at $[\mathrm{Fe} / \mathrm{H}]<-0.2$ there are only four planet hosts, so in the following, we focus on what we define as solar twins (see Table 2). In Fig. 7 we present the Li relation with mass for solar twins stars in two metallicity regions. In general, the stars are homogeneously spread at several masses regardless of their Li depletion at both metallicity ranges though the average mass of planet hosts is higher due to their higher average $[\mathrm{Fe} / \mathrm{H}]$. We can also observe that stars with similar masses in each metallicity region present a high dispersion in Li abundances. All the planet hosts with higher $\mathrm{Li}$ abundances are situated in the metal-rich panel $(0<[\mathrm{Fe} / \mathrm{H}]<0.2)$, but the average abundance of Li detections, $1.33 \mathrm{dex}$, is lower than for the single stars, 1.69. Even if we 


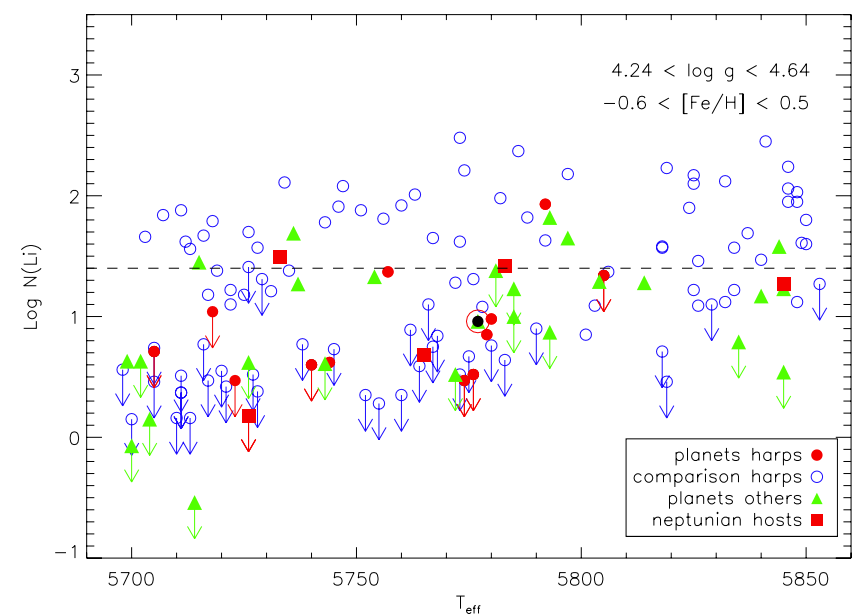

Fig. 5. Lithium abundances vs. $T_{\text {eff }}$ for solar analogues. Symbols as in Fig. 2.

remove the younger stars $(<1.5 \mathrm{Gyr})$, single stars have on average 0.30 dex higher abundances ( 1.33 vs. 1.63 , see Sect. 4.5 and Table 5 for further discussion about the $\mathrm{Li}$ dependence on age). For the lower metal-poor region $(-0.2<[\mathrm{Fe} / \mathrm{H}]<0)$, the difference is more obvious. Here we find that the average of $\mathrm{Li}$ detections for planet hosts is 1.02, whereas for single stars it is 1.79 . If we discard young stars $(<1.5 \mathrm{Gyr})$, the difference is practically the same. This result does not depend on stellar mass. We do not find any trend in $\mathrm{Li}$ abundances with this parameter, and the average masses of both samples are very similar (see Table 5).

This spread has been noticed in previous works, such as Pace et al. (2012), where they show how Li abundances do not depend on mass for solar type stars (one solar mass or lower) in M67, and they suggest that an extra variable, in addition to mass, age, and metallicity, is responsible for the scatter in $\mathrm{Li}$ abundances, since those stars are very similar. A similar spread in $\mathrm{Li}$ is observed for field stars around one solar mass and $-0.2<$ $[\mathrm{Fe} / \mathrm{H}]<0$ in Lambert \& Reddy (2004). Looking at these plots, it is very clear that planet hosts have destroyed more Li regardless of their $T_{\text {eff }}$, mass, or metallicity, so that another parameter must exist that explains this trend.

\subsection{Li and age}

Some previous works have claimed that the observed Li depletion in planet hosts is a consequence of the evolution of the star (e.g. Baumann et al. 2010) and thus depends on age. Therefore, if planet hosts were systematically older than single stars that might be the reason for their lower Li abundances. We want to emphasize that for stars with ages between 1.5 and $8 \mathrm{Gyr}$, the width of spectral lines do not change considerably since $v \sin i$ is nearly constant during MS (e.g. Pace \& Pasquini 2004). Therefore, for radial velocity surveys, these are all good targets for precise planet searches regardless of their age. We agree that $\mathrm{Li}$ is destroyed as the star gets older, but this depletion takes place principally during the first 1 Gyr (Randich 2010) (if we consider logarithmic abundances) and depends on initial rotation rates (e.g. Charbonnel \& Talon 2005), whereas after 1-2 Gyr the age effect is not so strong. For instance, the models by Deliyannis \& Pinsonneault (1997), which include rotational induced mixing, show $\mathrm{Li}$ abundances of $\sim 2.9$ dex, $\sim 2.1$ dex, and $\sim 1.7$ dex at ages of $100 \mathrm{Myr}, 1.7 \mathrm{Gyr}$, and $4 \mathrm{Gyr}$, respectively, for a star with $T_{\mathrm{eff}}=5800 \mathrm{~K}$ and initial rotation

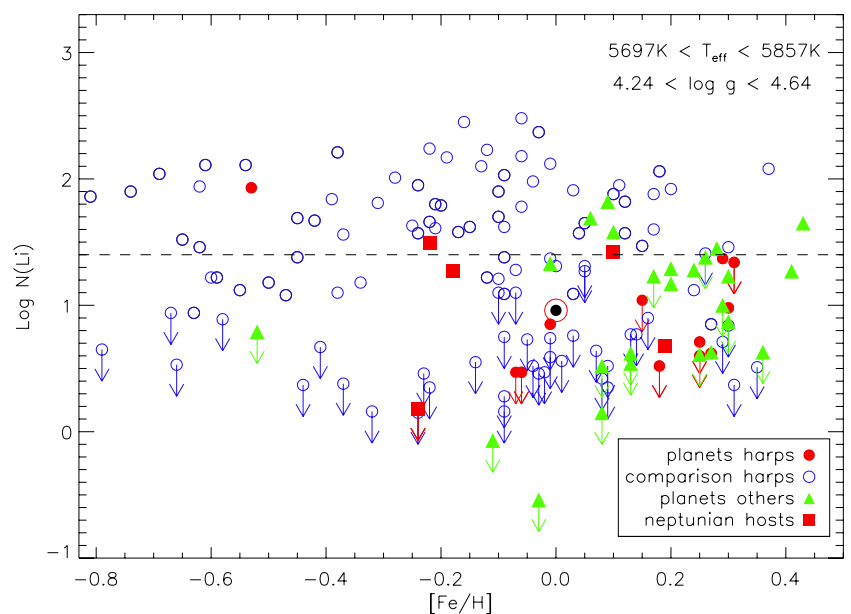

Fig. 6. Lithium abundances vs. $[\mathrm{Fe} / \mathrm{H}]$ for solar analogues. Symbols as in Fig. 2.

$v \sin i=10 \mathrm{~km} \mathrm{~s}^{-1}$. Furthermore, if age were the principal cause of Li depletion, we would not observe such a dispersion $(\sim 1.3 \mathrm{dex})$ in $\mathrm{Li}$ abundances for stars in the same evolutionary status in clusters like M67, where all the stars have the same age and metallicity (Pasquini et al. 2008; Randich et al. 2009; Pace et al. 2012). In the clusters NGC 3960 (Prisinzano \& Randich 2007), Collinder 261 (Pallavicini et al. 2005), and NGC 6253 (Randich 2010), a Li dispersion in solar type stars was found as well, though not as large as in M67. On the other hand, the near solar metallicity cluster NGC 188 of 6-8 Gyr present abundances 10-20 times higher than in the Sun (Randich et al. 2003).

In the left-hand panel of Fig. 8 we explore the relation of $\mathrm{Li}$ with age for solar twins. We observe that there is no a tight relation with age except for very young ages. Furthermore, we can find young stars with low $\mathrm{Li}$ abundances, as well as old stars with higher abundances. Both groups of stars are spread over the entire range of ages, though at younger ages ( $0-2$ Gyr) there are more single stars than planet hosts. This is likely due to the higher difficulties in finding planets around young, active stars. In order to test this correlation, we computed a generalized Kendall's tau correlation coefficient considering upper limits of $\mathrm{Li}$. This was done with the program $\mathrm{R}$ and the function cenken, which can be used with censored data (Akritas et al. 1995). This test gives $\tau=0.138$ (with a probability of 0.63 of having a correlation) for planet hosts and $\tau=-0.004$ for single stars (with $P=0.03$ of having a correlation). Therefore, this test shows that there is no a clear relationship between $\mathrm{Li}$ and age. However, if we only deal with the Li detections $\tau$ decreases to -0.24 for both groups, with a higher probability of correlation for single stars, 0.96 vs. 0.63 for planet hosts.

In the right-hand panel of Fig. 8 we can observe the same plot but split in two mass ranges, $0.89-1.0 M_{\odot}$ and 1.0-1.1 $M_{\odot}$. In the more massive subsample (red symbols) we can only find detections of Li for single stars at ages $<3 \mathrm{Gyr}$, so we cannot compare stars at older ages. However, in that age bin (0-3 Gyr), none of the planet hosts can reach the average of Li for single stars. On the other hand, the number of stars is higher in the less massive subgroup (purple symbols). Planet hosts are spread at different ages, but in general present lower Li abundances than the single stars of similar ages. In any case, the stars of both mass subgroups might not be very different considering typical errors in mass of $0.1 M_{\odot}$ (e.g. Casagrande et al. 2007; Fernandes et al. 2011). In addition, we show in the previous section that for solar twins $\left(0.9-1.1 M_{\odot}\right)$, Li does not depend on 

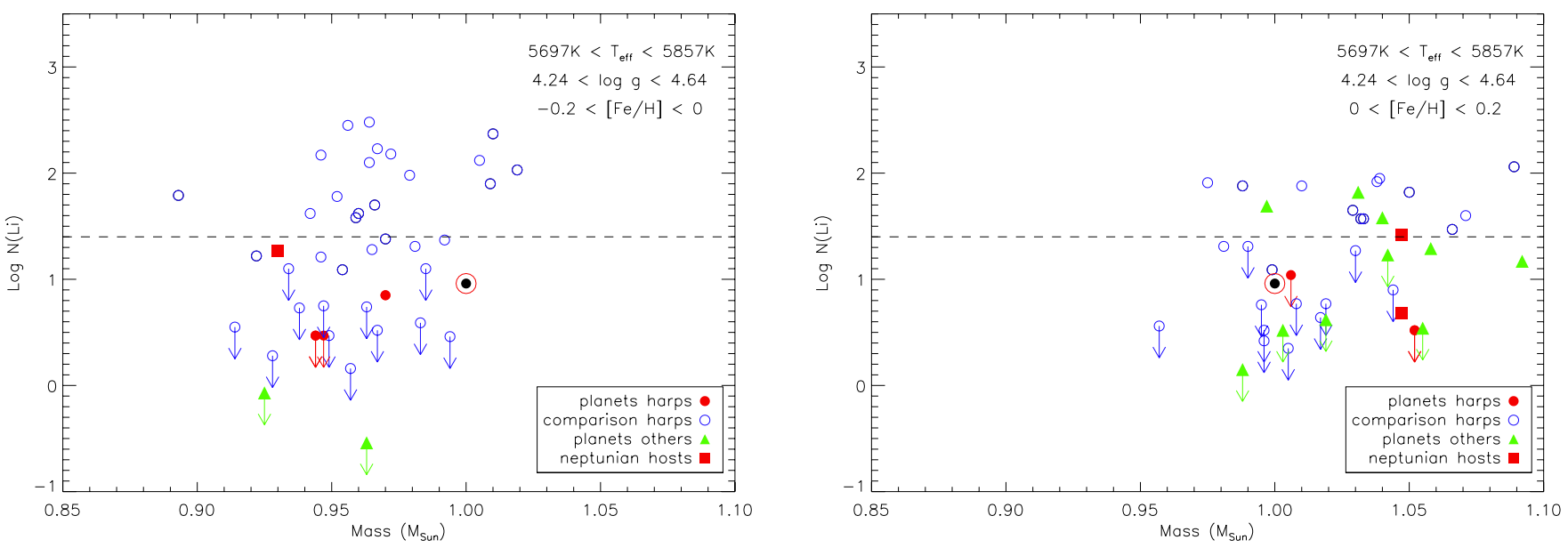

Fig. 7. Lithium abundances vs. mass for solar twins at $[\mathrm{Fe} / \mathrm{H}]<0$ (left panel) and solar twins at $[\mathrm{Fe} / \mathrm{H}]>0$ (right panel). Symbols as in Fig. 2.

Table 5. Mean values of parameters, together with standard error of the mean, for different subsamples, all of them with $T_{\text {eff }}=T_{\odot} \pm 80 \mathrm{~K}$, and $\log g=\log g_{\odot} \pm 0.2$.

\begin{tabular}{lrcccccccc}
\hline \hline Group & Number & {$[\mathrm{Fe} / \mathrm{H}]$ range } & $\begin{array}{c}\text { Age range } \\
\mathrm{Gyr}\end{array}$ & $\begin{array}{c}T_{\text {eff }} \\
(\mathrm{K})\end{array}$ & {$[\mathrm{Fe} / \mathrm{H}]$} & $\begin{array}{c}\log g \\
\left(\mathrm{~cm} \mathrm{~s}^{-2}\right)\end{array}$ & $\begin{array}{c}\text { Mass } \\
M_{\odot}\end{array}$ & $\begin{array}{c}\text { Age } \\
\mathrm{Gyr}\end{array}$ & $\begin{array}{c}\text { Li detections } \\
A(\mathrm{Li})\end{array}$ \\
\hline $\mathrm{A}$ & $10 \mathrm{PH}$ & {$[-0.2,0.2]$} & {$[1.5,12]$} & $5797 \pm 12$ & $0.07 \pm 0.04$ & $4.41 \pm 0.03$ & $1.02 \pm 0.01$ & $5.14 \pm 0.82$ & $1.27 \pm 0.12$ \\
$\mathrm{~A}$ & $30 \mathrm{CS}$ & {$[-0.2,0.2]$} & {$[1.5,12]$} & $5783 \pm 8$ & $-0.04 \pm 0.02$ & $4.47 \pm 0.02$ & $0.98 \pm 0.01$ & $4.01 \pm 0.44$ & $1.75 \pm 0.07$ \\
\hline $\mathrm{B}$ & $5 \mathrm{PH}$ & {$[-0.2,0.2]$} & {$[4,12]$} & $5805 \pm 15$ & $0.02 \pm 0.06$ & $4.36 \pm 0.03$ & $1.01 \pm 0.03$ & $7.03 \pm 1.04$ & $1.13 \pm 0.10$ \\
$\mathrm{~B}$ & $11 \mathrm{CS}$ & {$[-0.2,0.2]$} & {$[4,12]$} & $5768 \pm 15$ & $-0.07 \pm 0.03$ & $4.40 \pm 0.02$ & $0.96 \pm 0.01$ & $6.63 \pm 0.62$ & $1.48 \pm 0.10$ \\
\hline \hline $\mathrm{C}$ & $3 \mathrm{PH}$ & {$[-0.2,0]$} & {$[1.5,12]$} & $5800 \pm 22$ & $-0.06 \pm 0.06$ & $4.40 \pm 0.02$ & $0.97 \pm 0.02$ & $7.50 \pm 1.74$ & $1.03 \pm 0.12$ \\
$\mathrm{C}$ & $22 \mathrm{CS}$ & {$[-0.2,0]$} & {$[1.5,12]$} & $5778 \pm 9$ & $-0.09 \pm 0.01$ & $4.47 \pm 0.02$ & $0.96 \pm 0.01$ & $4.33 \pm 0.53$ & $1.77 \pm 0.09$ \\
\hline $\mathrm{D}$ & $3 \mathrm{PH}$ & {$[-0.2,0]$} & {$[4,12]$} & $5800 \pm 22$ & $-0.06 \pm 0.06$ & $4.40 \pm 0.02$ & $0.97 \pm 0.02$ & $7.50 \pm 1.74$ & $1.03 \pm 0.12$ \\
$\mathrm{D}$ & $9 \mathrm{CS}$ & {$[-0.2,0]$} & {$[4,12]$} & $5770 \pm 17$ & $-0.10 \pm 0.02$ & $4.41 \pm 0.03$ & $0.95 \pm 0.01$ & $6.72 \pm 0.76$ & $1.48 \pm 0.11$ \\
\hline \hline $\mathrm{E}$ & $8 \mathrm{PH}$ & {$[0,0.2]$} & {$[1.5,12]$} & $5793 \pm 13$ & $0.12 \pm 0.03$ & $4.42 \pm 0.03$ & $1.04 \pm 0.01$ & $4.17 \pm 0.58$ & $1.33 \pm 0.13$ \\
$\mathrm{E}$ & $9 \mathrm{CS}$ & {$[0,0.2]$} & {$[1.5,12]$} & $5795 \pm 16$ & $0.09 \pm 0.02$ & $4.45 \pm 0.03$ & $1.02 \pm 0.01$ & $3.58 \pm 0.10$ & $1.63 \pm 0.10$ \\
\hline $\mathrm{F}$ & $3 \mathrm{PH}$ & {$[0,0.2]$} & {$[4,12]$} & $5800 \pm 20$ & $0.10 \pm 0.06$ & $4.34 \pm 0.05$ & $1.05 \pm 0.02$ & $5.72 \pm 0.83$ & $1.18 \pm 0.13$ \\
$\mathrm{~F}$ & $3 \mathrm{CS}$ & {$[0,0.2]$} & {$[4,12]$} & $5763 \pm 27$ & $0.04 \pm 0.03$ & $4.36 \pm 0.02$ & $0.99 \pm 0.01$ & $6.54 \pm 0.34$ & $1.43 \pm 0.24$ \\
\hline \hline $\mathrm{G}$ & $8 \mathrm{PH}$ & {$[-0.25,0.15]$} & {$[1.5,12]$} & $5786 \pm 15$ & $-0.01 \pm 0.04$ & $4.44 \pm 0.03$ & $0.99 \pm 0.02$ & $5.85 \pm 1.07$ & $1.38 \pm 0.12$ \\
$\mathrm{G}$ & $35 \mathrm{CS}$ & {$[-0.25,0.15]$} & {$[1.5,12]$} & $5789 \pm 8$ & $-0.09 \pm 0.02$ & $4.46 \pm 0.02$ & $0.96 \pm 0.01$ & $4.52 \pm 0.50$ & $1.75 \pm 0.07$ \\
\hline $\mathrm{H}$ & $5 \mathrm{PH}$ & {$[-0.25,0.15]$} & {$[4,12]$} & $5783 \pm 18$ & $-0.06 \pm 0.06$ & $4.40 \pm 0.04$ & $0.97 \pm 0.03$ & $7.65 \pm 0.98$ & $1.20 \pm 0.13$ \\
$\mathrm{H}$ & $16 \mathrm{CS}$ & {$[-0.25,0.15]$} & {$[4,12]$} & $5779 \pm 14$ & $-0.12 \pm 0.03$ & $4.40 \pm 0.02$ & $0.95 \pm 0.01$ & $6.87 \pm 0.57$ & $1.53 \pm 0.07$ \\
\hline $\mathrm{I}$ & $4 \mathrm{PH}$ & {$[-0.25,0.15]$} & {$[5,12]$} & $5785 \pm 23$ & $4.39 \pm 0.05$ & $-0.08 \pm 0.08$ & $8.45 \pm 0.74$ & $0.96 \pm 0.03$ & $1.26 \pm 0.14$ \\
$\mathrm{I}$ & $12 \mathrm{CS}$ & {$[-0.25,0.15]$} & {$[5,12]$} & $5777 \pm 17$ & $4.38 \pm 0.02$ & $-0.12 \pm 0.03$ & $7.73 \pm 0.57$ & $0.94 \pm 0.01$ & $1.52 \pm 0.08$ \\
\hline
\end{tabular}

Notes. Metallicity and age ranges (which include the boundaries) are indicated in the table. PH and CS denote planet hosts (from HARPS and other spectrographs) and comparison sample stars, respectively.

mass, even in narrower metallicity ranges, so mixing all those in the same plot (left panel of Fig. 8) will not affect our conclusions. Furthermore, we note that when dealing with MS stars, the age determination is probably very uncertain (e.g. Jørgensen \& Lindegren 2005), at least significantly more uncertain than the mass determination.

Finally, in Fig. 9 we depict the average of Li detections for solar twins in different age bins. We can observe a decrease in $\mathrm{Li}$ abundances for solar age stars with respect to younger objects but stars with planets always present lower Li abundances in each age bin for ages $<8$ Gyr. At older ages, we only have three objects so we do not contemplate those age bins.

In Table 5 we summarize the average values of parameters in several subsamples already discussed. We note that Li averages are calculated with only detections since for upper limits we cannot know the real content of Li. We discard the younger stars $(<1.5 \mathrm{Gyr})$ to avoid a comparison sample that is too biased towards low ages. For the solar twins $(-0.2<[\mathrm{Fe} / \mathrm{H}]<0.2$, group $\mathrm{A}$ in the table) the single stars present $\mathrm{Li}$ abundances nearly 0.5 dex higher than planet hosts and only the planet host HD 9446 present a Li abundance higher than the average for single stars. This difference in mean $\mathrm{Li}$ is higher than $3.4 \sigma$ (two side t-test), whereas the difference for age is $1.2 \sigma$. For older stars (>4 Gyr, group B), the difference is also high, 0.35 dex, but in this case none of the planet hosts reach $A(\mathrm{Li})=1.48$. The planet host group has higher average $[\mathrm{Fe} / \mathrm{H}]$, but that difference in metallicity is not enough to explain the difference in $\mathrm{Li}$ as already discussed in previous sections. Moreover, Pinsonneault et al. (2001) show that the mass of the convective envelope (responsible for the degree of Li depletion) hardly varies for a wide range of metallicities at a given $T_{\text {eff }}$. Nevertheless, we find different $\mathrm{Li}$ abundances if we split the solar twins into two metallicity regions.

In the less metallic group $(-0.2<[\mathrm{Fe} / \mathrm{H}]<0)$, "single" stars are very biased towards young ages (group C) so we must only take older stars (group D) into account. For the more metallic 

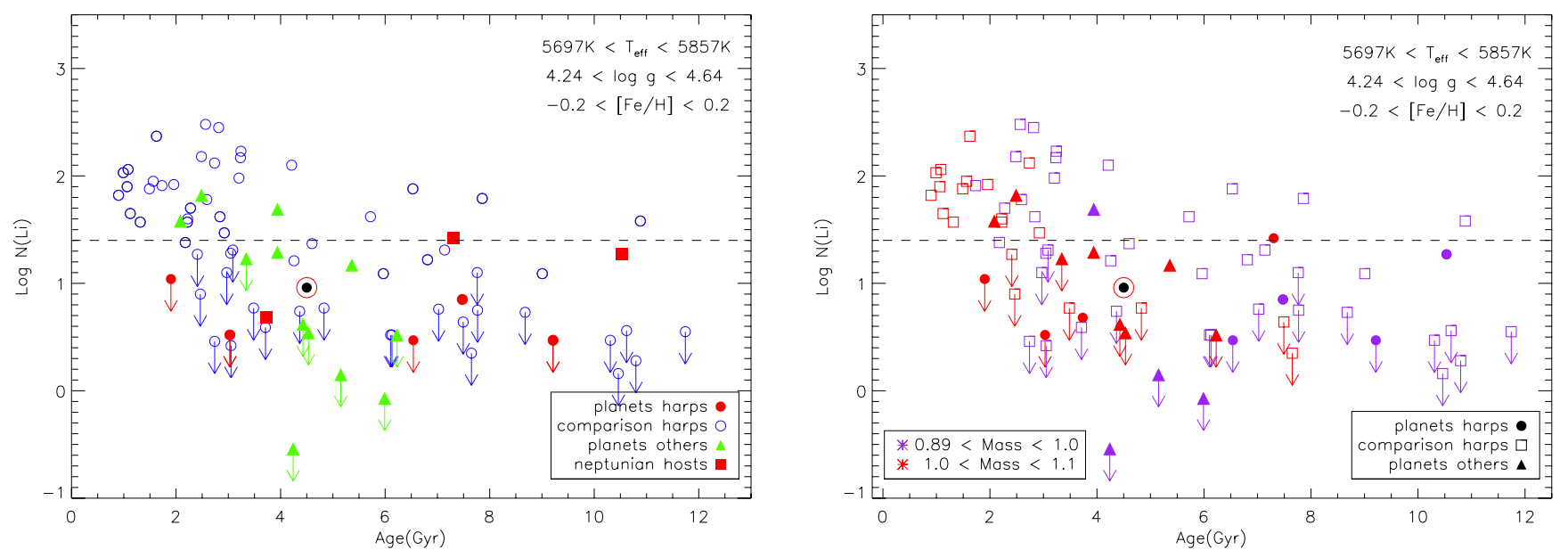

Fig. 8. Lithium abundances vs. age for solar twins (left panel) and for solar twins with colours denoting different mass ranges (right panel).

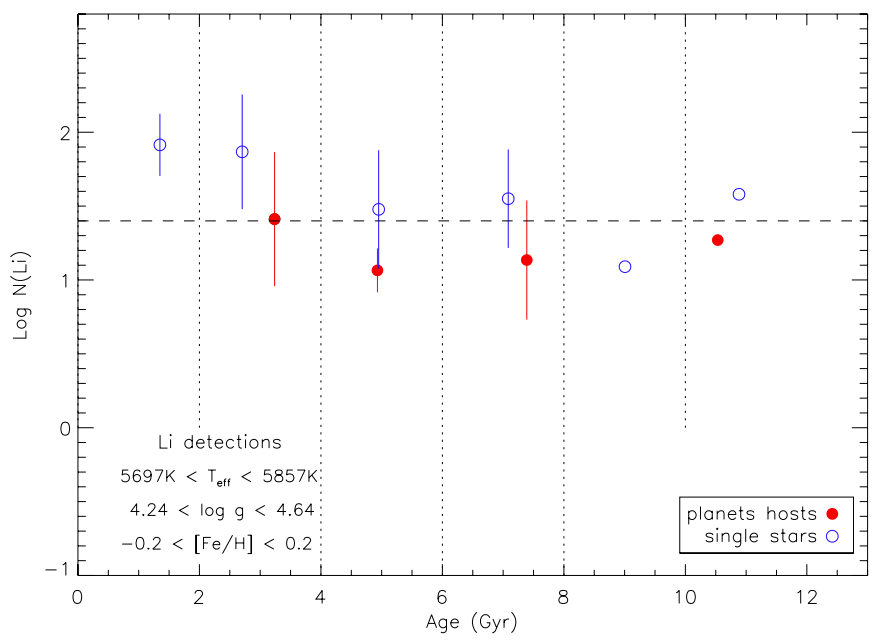

Fig. 9. Average of Li detections for solar twins in different age bins. The points in each bin are situated in their average age. The bars indicate the dispersion in $\mathrm{Li}$ abundances if there are more than one star in each bin.

stars (groups E and F), the mean ages and metallicities are more similar in the younger subsample (group E), and we find a difference of 0.3 dex in Li abundance. Planet hosts are also slightly older on average $(\sim 1 \mathrm{Gyr})$ in most of the subsamples, but the difference is at the level of the errors, and as we already pointed out, the main destruction of Li occurs before 1-2 Gyr. Since we are not considering those young objects, the slight difference in average ages cannot justify the big differences in Li. We also look at a subsample of solar twins shifted to lower metallicities $([\mathrm{Fe} / \mathrm{H}]=-0.05 \pm 0.2$, groups $\mathrm{G}$ and $\mathrm{H})$, and the difference in $\mathrm{Li}$ abundances is quite similar, 0.43 and 0.33 dex for the groups with ages $>1.5$ Gyr and $>4$ Gyr, respectively. In order to find more similar populations regarding age and metallicity we analysed an older subsample: group I. The differences in average $[\mathrm{Fe} / \mathrm{H}]$ and age are similar to group $\mathrm{H}, 0.04 \mathrm{dex}$, and $0.7 \mathrm{Gyr}$, respectively, while the $\mathrm{Li}$ difference is decreased to 0.26 dex. This suggests that for older ages, it is more difficult to detect differences, though the number of stars in this subsample is significantly lower and thus the statistical inferences might be less reliable.

With the aim of increasing the number of stars in our sample we applied survival statistics to several subsamples from Table 5, which allows information to be extracted from the upper limits.
We used the package ASURV (Feigelson \& Nelson 1985), which provides the Kaplan-Meier estimator of the mean and several two-sample tests. For the group A, four out of five tests give probabilities lower than 0.06 that the samples of planets hosts and "single" stars come from the same parent population. The Kaplan-Meier estimator of the mean is $0.361 \pm 0.208$ for planets hosts and $1.069 \pm 0.116$ for stars without planets, hence it seems these two samples are really distinct. For the older solar twins (group B), however all the two-sample tests give probabilities between 0.4-0.6. The K-M estimator of the mean for planet hosts is $0.104 \pm 0.229$ and for stars without detected planets it is $0.722 \pm 0.135$. Therefore for this subgroup there is no clear difference. For the solar twins sample at lower $[\mathrm{Fe} / \mathrm{H}]$ (group G), we find similar results as for group A, with probabilities around 0.08 that the samples are drawn from the same population. The K-M estimators of the mean are $0.330 \pm 0.233$ and $1.094 \pm 0.109$ for planet hosts and "single" stars, respectively, thus these subsamples appear to be different. For the older stars (group H), the two-sample test probabilities increase to 0.2 but the K-M estimators of the mean are still quite different $(0.081 \pm 0.226$ vs. $0.804 \pm 0.123)$. For the group I the two-sample test probabilities increase to 0.55 (and the K-M estimators of the mean are more similar) suggesting that the possible effect of planets might be diluted for very old stars as pointed out before.

These tests including upper limits and the values from detections shown in Table 5 indicate that there seems to be a different distribution of Li abundances for planet hosts. However, depending on the building of the subsamples and the restrictions applied to age and metallicity, the results can be different, maybe owing to the low number of stars in each subsample. Therefore, a bigger sample with more similar stars would be desirable to fully confirm this distinction.

\subsection{Testing the differences with observed spectra}

In Fig. 10 several pairs of stars with very similar parameters are depicted. The stars in each pair have maximum differences of $40 \mathrm{~K}, 0.06 \mathrm{dex}, 0.2 \mathrm{dex}, 0.06 M_{\odot}$, and $1 \mathrm{Gyr}$ for $T_{\text {eff }}$, [Fe/H], $\log g$, mass, and age, respectively. These plots are a definitive demonstration that other parameters are affecting Li depletion, otherwise we would not be able to explain the difference in $\mathrm{Li}$ line for these almost twins stars (in each pair). The difference between parameters is about $1 \sigma$, while difference in $\mathrm{Li}$ abundances goes from $0.3 \mathrm{dex}$ (more than $3 \sigma$ ) to more than $1.5 \mathrm{dex}$ 

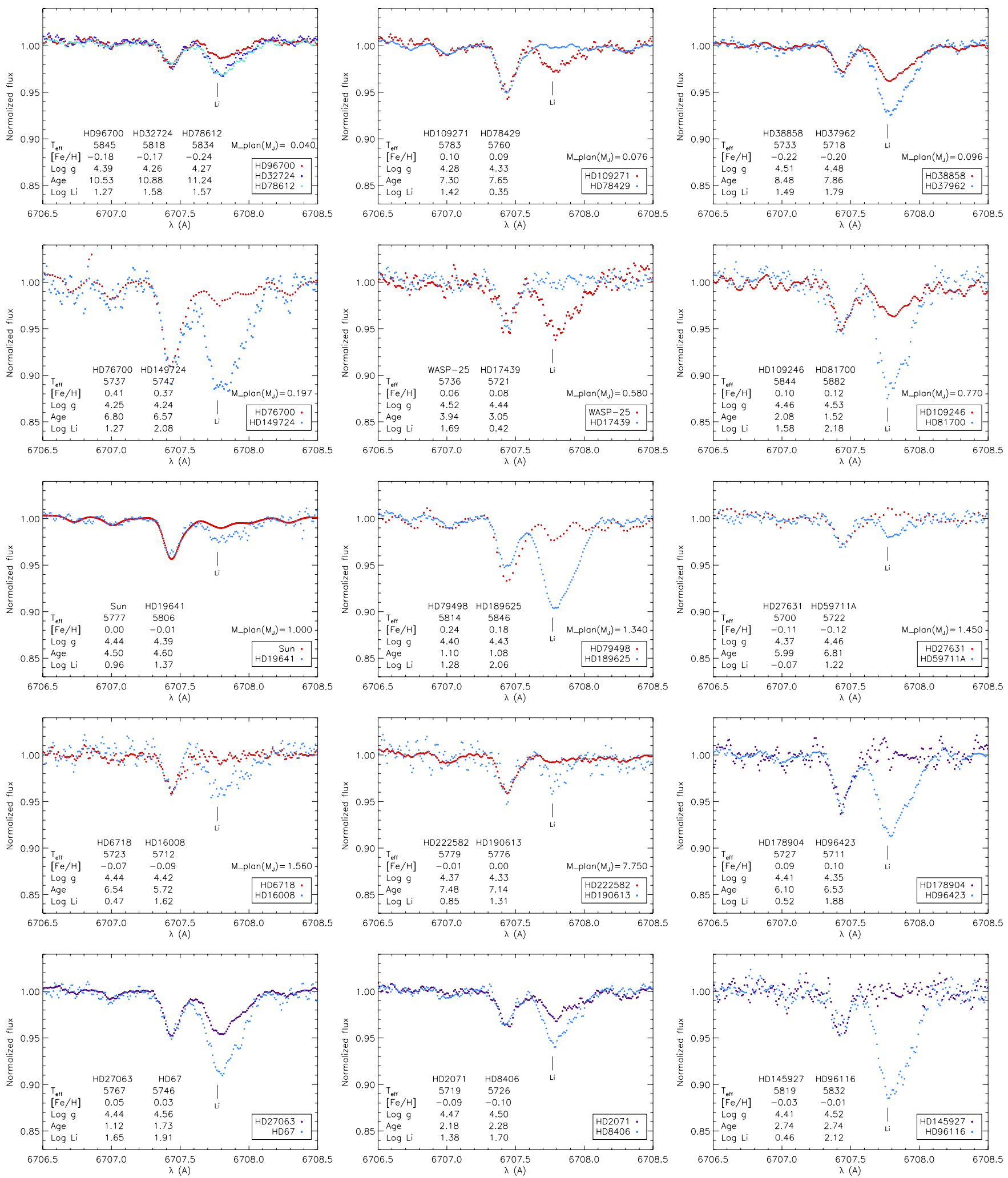

Fig. 10. Observed spectra for couples of stars with very similar parameters and different Li abundances. Planet hosts spectra are depicted with red dots and stars without known planets are depicted with blue or purple dots. For the couples with a planet host the minimum mass of the most massive planet in each system is indicated in the plot.

for the pair with HD 145927 and HD 96116 in which the two stars have the same age. We can find couples in which both stars do not have known planets (but present a quite distinct degree of Li depletion), and we can also find couples in which the planet host has a Li abundance higher than the single star. However, all those planet hosts (except HD 9446) have smaller planets (see next section). Indeed, it is easy to detect the Li absorption in some of those stars with smaller planets but when you move to 


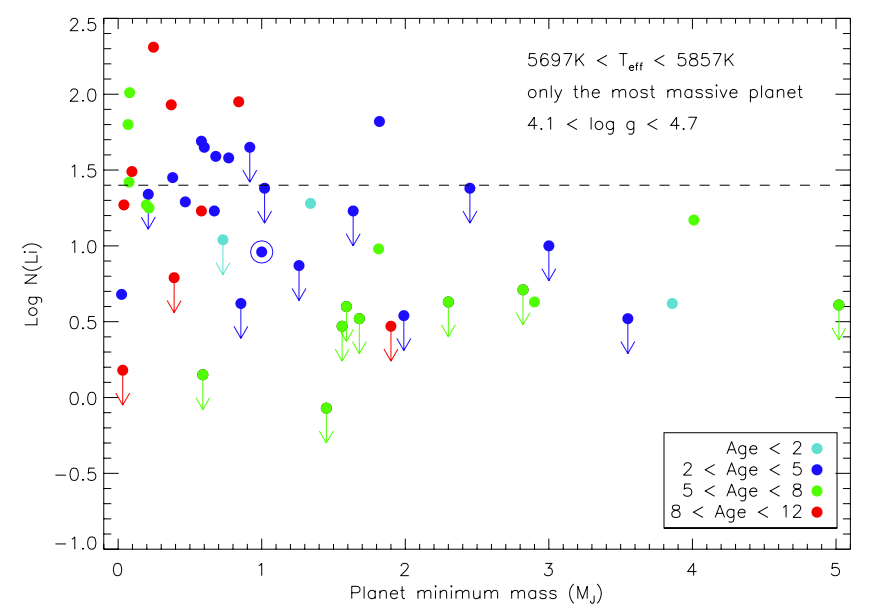

Fig. 11. Lithium abundances vs. minimum planetary masses for the most massive planet in each system. Colours denote different age ranges.

planetary masses higher than that of Jupiter, the Li line almost disappears. Regardless of the presence of planets, these huge differences in Li cannot be justified with the observed differences in stellar parameters.

Consequently, we can conclude that neither mass, age, nor metallicity can be the only cause of the extra Li depletion for our solar analogues. Other mechanisms such as rotationally induced mixing or overshooting mixing should be considered.

\subsection{Li and planetary parameters}

We also searched for any possible relation of previously shown $\mathrm{Li}$ depletion with the physical and orbital parameters of the planets, that is, mass, period, eccentricity and semi-major axis. We did not find any dependence except possibly on the planetary mass. In Fig. 11 we plot the Li abundances as a function of the more massive planet in each system. We note that using the sum of masses of all the planets in each system produces a similar plot. It seems that the destruction of $\mathrm{Li}$ is higher when the planet is more massive. This would make sense in a scenario where the disc is affecting the evolution of angular momentum, hence mixing mechanisms (Bouvier 2008), since we could expect a stronger effect if the disc is more massive and has a longer lifetime, conditions needed to form a giant planet. In fact, all the stars with planets more massive than Jupiter (except HD 9446) have depleted their Li significantly. Furthermore, the accretion processes are expected to be more frequent and violent when there is a giant planet in the disc and, as a consequence, produce $\mathrm{Li}$ destruction either by the increase in temperature on the base of the convective envelope (Baraffe \& Chabrier 2010) or by extra-mixing triggered by thermohaline convection (Théado \& Vauclair 2012).

In Fig. 11 we only constrain the $T_{\text {eff }}$ of the stars but not their $\log g$ or $[\mathrm{Fe} / \mathrm{H}]$ so some of the planet hosts with higher Li abundances have low $\log g$ and might be slightly evolved. In fact, the planet hosts with higher Li detections are older than $5 \mathrm{Gyr}$, and half of them are older than 8 Gyr. As discussed in previous sections this might be the reason for the high $\mathrm{Li}$ abundance and not the mass of the planet, but what is certain is that all the stars with planets within solar $\log g$ range and high Li abundances host less massive planets. Therefore, the mass of the disc in those systems would not be enough to affect the stellar angular momentum evolution or to produce intense accretion episodes and thus, those stars would present a different degree of Li depletion depending on other parameters. We could say that those planet hosts behave in a similar way to stars without planets, since some of them have destroyed their Li, but others have not and the mechanism responsible for depleting it should be other than the presence of planets. We should also take into account that most of the $\mathrm{Li}$ abundances are upper limits so we can just conclude that $\mathrm{Li}$ is severely depleted at least when the planet is massive enough. To extract the information of upper limits, we computed the Kendall's tau correlation coefficient for censored data as done before. This test gives $\tau=0.016$ (with $P=0.12$ ), which means there is no correlation. If we only consider the detections, $\tau$ decreases to -0.215 (with $P=0.87$ ), pointing to a possible correlation, but the result is not significant enough. Nevertheless, we think that this plot shows an interesting relation and should be considered in further studies of $\mathrm{Li}$ abundances when the number of low-mass planet hosts increases.

\section{Conclusions}

We have presented $\mathrm{Li}$ abundances in a large sample of planet hosts and single stars in the effective temperature range $5600 \mathrm{~K}<$ $T_{\text {eff }}<5900 \mathrm{~K} .42$ planet hosts and 235 single stars come from HARPS GTO sample, while 49 other planet hosts have been observed with other instruments. We find that the average Li abundance for single stars is greater than for the planet hosts. This difference is more obvious when we look at solar twins with $T_{\text {eff }}=$ $T_{\odot} \pm 80 \mathrm{~K}, \log g=\log g_{\odot} \pm 0.2$, and $[\mathrm{Fe} / \mathrm{H}]=[\mathrm{Fe} / \mathrm{H}]_{\odot} \pm 0.2$ where only $18 \%$ of planet hosts show detections of $A(\mathrm{Li})>1.4$ whereas for single stars this value reaches $48 \%$ confirming previous studies (e.g. Israelian et al. 2009; Gonzalez et al. 2010; Takeda et al. 2010; Sousa et al. 2010).

We checked whether our abundances depend on other parameters, such as mass, age, or metallicity. We find that most of our stars at very high metallicities present low Li content regardless of the presence of planets. On the other hand, if planet hosts were much older than the single stars, we might expect that to be the reason of the extra depletion since Li shows a small decrease with age as observed in open clusters of several ages (Sestito \& Randich 2005). After removing the younger objects (age $<1.5 \mathrm{Gyr}$ ), we showed that planet hosts are around $1 \mathrm{Gyr}$ older on average, but this small age difference cannot be responsible for the large difference in $\mathrm{Li}$ abundances (more than $3 \sigma$ ) found between stars with and without planets. However, some statistical tests, including the $\mathrm{Li}$ upper limits, show that both populations might be not so different depending on the ranges in age and $[\mathrm{Fe} / \mathrm{H}]$ used to define the subsamples. On the other hand, we show several examples of couples formed by twins with huge differences in abundance, up to 1.6 dex. Therefore, we suggest that the presence of giant planets (among other possible causes) might affect the Li content in solar twins.

The dispersion of $\mathrm{Li}$ abundances in stars of similar $T_{\mathrm{eff}}$, mass, age, and metallicity have also been reported in several open clusters like M67, which represents a good comparison for our observation since it has solar age and metallicity. Moreover, the standard model for stellar evolution, which considers only convection as a mixing mechanism, cannot explain the extra $\mathrm{Li}$ depletion found in old stars (regardless of the presence of planets) during the MS. Therefore, there must be non-standard processes that produce this effect, so we propose that the presence of planets is one of them. For instance, the stars with low Li in M67 might have planets that have not been discovered yet. Indeed, two of the planet candidates proposed in this cluster by Pasquini et al. (2012) have $T_{\text {eff }}$ in our solar range and present 
low Li abundances (Pasquini et al. 2008). However we also find stars without detected planets which are heavily depleted in $\mathrm{Li}$; as a result other mechanisms such as rotationally induced mixing (Pinsonneault et al. 1990) must also be considered. In fact, this kind of mixing is related to the initial rotation rates and angular momentum evolution of the star and thus has a strong connection with protoplanetary discs. Therefore, the presence of a planetary disc may be one of the causes to produce this kind of mixing. In addition, planets can also cause an extra Li depletion through other mechanisms such as violent accretion-burst episodes of planetary material. Both mechanisms produce a stronger effect when the protoplanetary discs are more massive, in agreement with our finding that $\mathrm{Li}$ is always destroyed if the star hosts a giant planet.

Acknowledgements. E.D.M., S.G.S., and V.Zh.A. acknowledge the support from the Fundação para a Ciência e Tecnologia, FCT (Portugal) in the form of the fellowships SFRH/BPD/76606/2011, SFRH/BPD/47611/2008, and SFRH/BPD/70574/2010 from the FCT (Portugal), respectively. G.I. and J.I.G.H. acknowledge financial support from the Spanish Ministry project MINECO AYA2011-29060, and J.I.G.H. also from the Spanish Ministry of Economy and Competitiveness (MINECO) under the 2011 Severo Ochoa Program MINECO SEV-2011-0187. E.D.M, S.G.S., N.C.S., A.M., and V.Zh.A. are grateful for the support by the European Research Council/European Community under the FP7 through Starting Grant agreement number 239953, as well as the support through programme Ciência 2007 funded by FCT/MCTES (Portugal) and POPH/FSE (EC), and in the form of grant PTDC/CTE-AST/098528/2008. We thank Pedro Figueira and the referee for useful comments and suggestions that helped improve the paper. This research has made use of the SIMBAD database operated at the CDS, Strasbourg (France) and the Encyclopaedia of Extrasolar Planets. This work has also made use of the IRAF facility.

\section{References}

Adibekyan, V. Z., Delgado Mena, E., Sousa, S. G., et al. 2012a, A\&A, 547, A36 Adibekyan, V. Z., Santos, N. C., Sousa, S. G., et al. 2012b, A\&A, 543, A89 Akritas, M. G., Murphy, S. A., \& LaValley, M. P. 1995, J. Am. Stat. Assoc., 90, 170

Alonso, R., Auvergne, M., Baglin, A., et al. 2008, A\&A, 482, L21

Ammler-von Eiff, M., Santos, N. C., Sousa, S. G., et al. 2009, A\&A, 507, 523

Arriagada, P., Butler, R. P., Minniti, D., et al. 2010, ApJ, 711, 1229

Baraffe, I., \& Chabrier, G. 2010, A\&A, 521, A44

Baumann, P., Ramírez, I., Meléndez, J., Asplund, M., \& Lind, K. 2010, A\&A, 519, A87

Bertelli, G., Girardi, L., Marigo, P., \& Nasi, E. 2008, A\&A, 484, 815

Boisse, I., Eggenberger, A., Santos, N. C., et al. 2010, A\&A, 523, A88

Bonomo, A. S., Hébrard, G., Santerne, A., et al. 2012, A\&A, 538, A96

Bouvier, J. 2008, A\&A, 489, L53

Casagrande, L., Flynn, C., Portinari, L., Girardi, L., \& Jimenez, R. 2007, MNRAS, 382, 1516

Castro, M., Vauclair, S., Richard, O., \& Santos, N. C. 2009, A\&A, 494, 663

Chaboyer, B., Demarque, P., \& Pinsonneault, M. H. 1995, ApJ, 441, 865

Charbonnel, C., \& Talon, S. 2005, Science, 309, 2189

Chen, Y. Q., \& Zhao, G. 2006, AJ, 131, 1816

Christensen-Dalsgaard, J., Gough, D. O., \& Thompson, M. J. 1991, ApJ, 378, 413

da Silva, R., Udry, S., Bouchy, F., et al. 2006, A\&A, 446, 717

Deliyannis, C. P., \& Pinsonneault, M. H. 1997, ApJ, 488, 836

Deliyannis, C. P., Demarque, P., \& Kawaler, S. D. 1990, ApJS, 73, 21

Eggenberger, P., Maeder, A., \& Meynet, G. 2010, A\&A, 519, L2

Eggenberger, P., Haemmerlé, L., Meynet, G., \& Maeder, A. 2012, A\&A, 539, A70

Feigelson, E. D., \& Nelson, P. I. 1985, ApJ, 293, 192

Fernandes, J. M., Vaz, A. I. F., \& Vicente, L. N. 2011, A\&A, 532, A20

Fischer, D. A., \& Valenti, J. 2005, ApJ, 622, 1102

Fischer, D. A., Marcy, G. W., Butler, R. P., et al. 2001, ApJ, 551, 1107

Garaud, P. 2011, ApJ, 728, L30

Garcia Lopez, R. J., Rebolo, R., \& Martin, E. L. 1994, A\&A, 282, 518

Ghezzi, L., Cunha, K., Smith, V. V., et al. 2009, ApJ, 698, 451

Ghezzi, L., Cunha, K., Smith, V. V., \& de la Reza, R. 2010, ApJ, 724, 154

Girardi, L., Bressan, A., Bertelli, G., \& Chiosi, C. 2000, A\&AS, 141, 371
Gonzalez, G. 1997, MNRAS, 285, 403

Gonzalez, G. 2008, MNRAS, 386, 928

Gonzalez, G., \& Laws, C. 2000, AJ, 119, 390

Gonzalez, G., Carlson, M. K., \& Tobin, R. W. 2010, MNRAS, 403, 1368

Hébrard, G., Bonfils, X., Ségransan, D., et al. 2010, A\&A, 513, A69

Israelian, G., Santos, N. C., Mayor, M., \& Rebolo, R. 2004, A\&A, 414, 601

Israelian, G., Delgado Mena, E., Santos, N. C., et al. 2009, Nature, 462, 189

Jørgensen, B. R., \& Lindegren, L. 2005, A\&A, 436, 127

King, J. R., Deliyannis, C. P., Hiltgen, D. D., et al. 1997, AJ, 113, 1871

Kurucz, R. 1993, ATLAS9 Stellar Atmosphere Programs and $2 \mathrm{~km} \mathrm{~s}^{-1}$ grid, Kurucz CD-ROM No. 13 (Cambridge, Mass.: Smithsonian Astrophysical Observatory)

Kurucz, R. L., Furenlid, I., Brault, J., \& Testerman, L. 1984, Solar flux atlas from 296 to $1300 \mathrm{~nm}$

Lambert, D. L., \& Reddy, B. E. 2004, MNRAS, 349, 757

Lo Curto, G., Mayor, M., Benz, W., et al. 2010, A\&A, 512, A48

Luck, R. E., \& Heiter, U. 2006, AJ, 131, 3069

Mayor, M., Pepe, F., Queloz, D., et al. 2003, The Messenger, 114, 20

Melo, C., Santos, N. C., Gieren, W., et al. 2007, A\&A, 467, 721

Minniti, D., Butler, R. P., López-Morales, M., et al. 2009, ApJ, 693, 1424

Montalban, J., \& Schatzman, E. 1996, A\&A, 305, 513

Montalbán, J., \& Schatzman, E. 2000, A\&A, 354, 943

Mortier, A., Santos, N. C., Sousa, S. G., et al. 2013, A\&A, 558, A106

Pace, G., Castro, M., Meléndez, J., Théado, S., \& do Nascimento, Jr., J.-D. 2012, A\&A, 541, A150

Pace, G., \& Pasquini, L. 2004, A\&A, 426, 1021

Pallavicini, R., Randich, S., \& Sestito, P. 2005, in 13th Cambridge Workshop on Cool Stars, Stellar Systems and the Sun, eds. F. Favata, G. A. J. Hussain, \& B. Battrick, ESA SP, 560, 867

Pasquini, L., Biazzo, K., Bonifacio, P., Randich, S., \& Bedin, L. R. 2008, A\&A, 489,677

Pasquini, L., Brucalassi, A., Ruiz, M. T., et al. 2012, A\&A, 545, A139

Pinsonneault, M. 1997, ARA\&A, 35, 557

Pinsonneault, M. H., Kawaler, S. D., \& Demarque, P. 1990, ApJS, 74, 501

Pinsonneault, M. H., Deliyannis, C. P., \& Demarque, P. 1992, ApJS, 78, 179

Pinsonneault, M. H., DePoy, D. L., \& Coffee, M. 2001, ApJ, 556, L59

Prisinzano, L., \& Randich, S. 2007, A\&A, 475, 539

Ramírez, I., Fish, J. R., Lambert, D. L., \& Allende Prieto, C. 2012, ApJ, 756, 46

Randich, S. 2010, in IAU Symp. 268, eds. C. Charbonnel, M. Tosi, F. Primas, \& C. Chiappini, 275

Randich, S., Aharpour, N., Pallavicini, R., Prosser, C. F., \& Stauffer, J. R. 1997, A\&A, 323, 86

Randich, S., Sestito, P., \& Pallavicini, R. 2003, A\&A, 399, 133

Randich, S., Primas, F., Pasquini, L., Sestito, P., \& Pallavicini, R. 2007, A\&A, 469, 163

Randich, S., Pace, G., Pastori, L., \& Bragaglia, A. 2009, A\&A, 496, 441

Richer, J., \& Michaud, G. 1993, ApJ, 416, 312

Ryan, S. G. 2000, MNRAS, 316, L35

Santos, N. C., Israelian, G., \& Mayor, M. 2004, A\&A, 415, 1153

Santos, N. C., Israelian, G., Mayor, M., et al. 2005, A\&A, 437, 1127

Santos, N. C., Mayor, M., Bonfils, X., et al. 2011, A\&A, 526, A112

Santos, N. C., Sousa, S. G., Mortier, A., et al. 2013, A\&A, 556, A150

Schneider, J., Dedieu, C., Le Sidaner, P., Savalle, R., \& Zolotukhin, I. 2011 A\&A, 532, A79

Sestito, P., \& Randich, S. 2005, A\&A, 442, 615

Setiawan, J., Weise, P., Henning, T., et al. 2007, ApJ, 660, L145

Sneden, C. A. 1973, Ph.D. Thesis, The University of Texas at Austin

Soderblom, D. R., Jones, B. F., Balachandran, S., et al. 1993, AJ, 106, 1059

Sousa, S. G., Santos, N. C., Israelian, G., Mayor, M., \& Monteiro, M. J. P. F. G. 2006, A\&A, 458, 873

Sousa, S. G., Santos, N. C., Mayor, M., et al. 2008, A\&A, 487, 373

Sousa, S. G., Fernandes, J., Israelian, G., \& Santos, N. C. 2010, A\&A, 512, L5

Sousa, S. G., Santos, N. C., Israelian, G., et al. 2011a, A\&A, 526, A99

Sousa, S. G., Santos, N. C., Israelian, G., Mayor, M., \& Udry, S. 2011b, A\&A, 533, A141

Sozzetti, A., Udry, S., Zucker, S., et al. 2006, A\&A, 449, 417

Takeda, Y., \& Kawanomoto, S. 2005, PASJ, 57, 45

Takeda, Y., Honda, S., Kawanomoto, S., Ando, H., \& Sakurai, T. 2010, A\&A, 515, A93

Tamuz, O., Ségransan, D., Udry, S., et al. 2008, A\&A, 480, L33

Théado, S., \& Vauclair, S. 2012, ApJ, 744, 123

Udry, S., \& Santos, N. C. 2007, ARA\&A, 45, 397

Udry, S., Mayor, M., Naef, D., et al. 2000, A\&A, 356, 590

Xiong, D. R., \& Deng, L. 2009, MNRAS, 395, 2013

Zahn, J.-P. 1992, A\&A, 265, 115

Zhang, Q. S. 2012, MNRAS, 427, 1441 
A\&A 562, A92 (2014)

Table 6. Li abundances for stars without detected planets from HARPS GTO samples. Parameters from Sousa et al. (2008, 2011a,b)

\begin{tabular}{|c|c|c|c|c|c|c|c|c|}
\hline Star & $\begin{array}{l}T_{\text {eff }} \\
(\mathrm{K})\end{array}$ & $\begin{array}{c}\log g \\
\left(\mathrm{~cm} \mathrm{~s}^{-2}\right)\end{array}$ & $\begin{array}{c}\xi_{\mathrm{t}} \\
\left(\mathrm{km} \mathrm{s}^{-1}\right)\end{array}$ & {$[\mathrm{Fe} / \mathrm{H}]$} & $\begin{array}{l}\text { Age } \\
(\mathrm{Gyr})\end{array}$ & $\begin{array}{l}\text { Mass } \\
\left(M_{\odot}\right)\end{array}$ & $A(\mathrm{Li})$ & Error \\
\hline \multicolumn{9}{|l|}{ HARPS-1 } \\
\hline HD 1320 & 5679 & 4.49 & 0.85 & -0.27 & 7.76 & 0.86 & 1.29 & 0.06 \\
\hline HD 2071 & 5719 & 4.47 & 0.95 & -0.09 & 2.18 & 0.97 & 1.38 & 0.07 \\
\hline HD 4307 & 5812 & 4.10 & 1.22 & -0.23 & 8.68 & 1.03 & 2.39 & 0.04 \\
\hline HD 4915 & 5658 & 4.52 & 0.90 & -0.21 & 3.12 & 0.91 & 1.38 & 0.05 \\
\hline HD 8406 & 5726 & 4.50 & 0.87 & -0.10 & 2.28 & 0.97 & 1.70 & 0.05 \\
\hline HD 11505 & 5752 & 4.38 & 0.99 & -0.22 & 11.43 & 0.88 & $<0.35$ & - \\
\hline HD 12387 & 5700 & 4.39 & 0.93 & -0.24 & 11.56 & 0.86 & $<0.15$ & - \\
\hline HD 13724 & 5868 & 4.52 & 1.02 & 0.23 & 0.82 & 1.10 & 1.60 & 0.05 \\
\hline HD 19467 & 5720 & 4.31 & 0.96 & -0.14 & 11.74 & 0.91 & $<0.55$ & - \\
\hline HD 20407 & 5866 & 4.50 & 1.09 & -0.44 & 11.34 & 0.84 & 1.85 & 0.05 \\
\hline HD 20619 & 5703 & 4.51 & 0.92 & -0.22 & 6.84 & 0.89 & 1.66 & 0.04 \\
\hline HD 20807 & 5866 & 4.52 & 1.04 & -0.23 & 6.43 & 0.94 & $<0.21$ & - \\
\hline HD 21938 & 5778 & 4.38 & 0.99 & -0.47 & 11.50 & 0.82 & 1.08 & 0.06 \\
\hline HD 27063 & 5767 & 4.44 & 0.94 & 0.05 & 1.12 & 1.03 & 1.65 & 0.05 \\
\hline HD 28471 & 5745 & 4.37 & 0.95 & -0.05 & 8.68 & 0.94 & $<0.73$ & - \\
\hline HD 28701 & 5710 & 4.41 & 0.95 & -0.32 & 11.60 & 0.84 & $<0.16$ & - \\
\hline HD 28821 & 5660 & 4.38 & 0.88 & -0.12 & 11.06 & 0.88 & $<0.11$ & - \\
\hline HD 32724 & 5818 & 4.26 & 1.14 & -0.17 & 10.88 & 0.96 & 1.58 & 0.05 \\
\hline HD 34449 & 5848 & 4.50 & 0.92 & -0.09 & 0.99 & 1.02 & 2.03 & 0.05 \\
\hline HD 37962 & 5718 & 4.48 & 0.84 & -0.20 & 7.86 & 0.89 & 1.79 & 0.05 \\
\hline HD 38277 & 5871 & 4.34 & 1.10 & -0.07 & 8.28 & 1.01 & 1.58 & 0.07 \\
\hline HD 44420 & 5818 & 4.37 & 1.06 & 0.29 & 2.25 & 1.08 & $<0.71$ & - \\
\hline HD 44594 & 5840 & 4.38 & 1.06 & 0.15 & 2.92 & 1.07 & 1.47 & 0.08 \\
\hline HD 45289 & 5717 & 4.32 & 0.99 & -0.02 & 10.31 & 0.95 & $<0.47$ & - \\
\hline HD 50806 & 5633 & 4.11 & 1.03 & 0.03 & 9.64 & 1.01 & 0.60 & 0.10 \\
\hline HD 59468 & 5618 & 4.39 & 0.88 & 0.03 & 8.44 & 0.93 & $<0.27$ & - \\
\hline HD 59711A & 5722 & 4.46 & 0.86 & -0.12 & 6.81 & 0.92 & 1.22 & 0.10 \\
\hline HD 66221 & 5635 & 4.40 & 0.92 & 0.17 & 3.74 & 1.00 & $<0.50$ & - \\
\hline HD 67458 & 5891 & 4.53 & 1.04 & -0.16 & 3.80 & 0.99 & 2.14 & 0.04 \\
\hline HD 71334 & 5694 & 4.37 & 0.95 & -0.09 & 9.19 & 0.91 & $<0.65$ & - \\
\hline HD 72769 & 5640 & 4.35 & 0.98 & 0.30 & 5.86 & 1.00 & $<0.51$ & - \\
\hline HD 76151 & 5788 & 4.48 & 0.96 & 0.12 & 0.90 & 1.05 & 1.82 & 0.05 \\
\hline HD 78429 & 5760 & 4.33 & 1.01 & 0.09 & 7.65 & 1.00 & $<0.35$ & - \\
\hline HD 78538 & 5786 & 4.50 & 0.98 & -0.03 & 1.62 & 1.01 & 2.37 & 0.05 \\
\hline HD 78558 & 5711 & 4.36 & 0.99 & -0.44 & 11.73 & 0.92 & $<0.37$ & - \\
\hline HD 78612 & 5834 & 4.27 & 1.14 & -0.24 & 11.24 & 0.94 & 1.57 & 0.05 \\
\hline HD 78747 & 5788 & 4.44 & 1.10 & -0.67 & 11.32 & 0.81 & $<0.94$ & - \\
\hline HD 88084 & 5766 & 4.42 & 0.96 & -0.10 & 7.76 & 0.93 & $<1.10$ & - \\
\hline HD 88218 & 5878 & 4.16 & 1.23 & -0.14 & 8.35 & 1.05 & 2.42 & 0.05 \\
\hline HD 89454 & 5728 & 4.47 & 0.96 & 0.12 & 1.32 & 1.03 & 1.57 & 0.07 \\
\hline HD 92719 & 5824 & 4.51 & 0.96 & -0.10 & 1.06 & 1.01 & 1.90 & 0.04 \\
\hline HD 95521 & 5773 & 4.49 & 0.96 & -0.15 & 2.84 & 0.96 & 1.62 & 0.05 \\
\hline HD 96423 & 5711 & 4.35 & 0.98 & 0.10 & 6.53 & 0.99 & 1.88 & 0.04 \\
\hline HD 97037 & 5883 & 4.34 & 1.13 & -0.07 & 7.89 & 1.00 & 1.65 & 0.05 \\
\hline HD 97998 & 5716 & 4.57 & 0.85 & -0.42 & 9.50 & 0.82 & 1.67 & 0.05 \\
\hline HD 104982 & 5692 & 4.44 & 0.91 & -0.19 & 8.64 & 0.88 & $<0.14$ & - \\
\hline HD 106116 & 5680 & 4.39 & 0.91 & 0.14 & 5.26 & 1.00 & $<0.13$ & - \\
\hline HD 108309 & 5775 & 4.23 & 1.08 & 0.12 & 7.59 & 1.04 & 0.94 & 0.10 \\
\hline HD 109409 & 5886 & 4.16 & 1.24 & 0.33 & 4.06 & 1.24 & 2.50 & 0.02 \\
\hline HD 110619 & 5613 & 4.51 & 0.83 & -0.41 & 6.33 & 0.82 & 1.11 & 0.10 \\
\hline HD 111031 & 5801 & 4.39 & 1.05 & 0.27 & 4.49 & 1.06 & 0.85 & 0.10 \\
\hline HD 114613 & 5729 & 3.97 & 1.18 & 0.19 & 4.50 & 1.24 & 2.63 & 0.03 \\
\hline HD 114853 & 5705 & 4.44 & 0.92 & -0.23 & 10.51 & 0.87 & $<0.46$ & - \\
\hline HD 115585 & 5711 & 4.27 & 1.14 & 0.35 & 7.48 & 1.04 & $<0.51$ & - \\
\hline HD 115674 & 5649 & 4.48 & 0.85 & -0.17 & 3.64 & 0.91 & $<0.40$ & - \\
\hline HD 117105 & 5889 & 4.41 & 1.13 & -0.29 & 11.12 & 0.90 & 1.93 & 0.05 \\
\hline HD 125184 & 5680 & 4.10 & 1.13 & 0.27 & 7.33 & 1.08 & $<0.39$ & - \\
\hline HD 134664 & 5865 & 4.52 & 0.99 & 0.10 & 1.10 & 1.07 & 2.10 & 0.05 \\
\hline HD 140901 & 5610 & 4.46 & 0.90 & 0.09 & 2.31 & 0.98 & $<0.48$ & - \\
\hline HD 143114 & 5775 & 4.39 & 0.92 & -0.41 & 11.58 & 0.84 & $<0.67$ & - \\
\hline HD 145809 & 5778 & 4.15 & 1.14 & -0.25 & 9.49 & 1.00 & 2.08 & 0.05 \\
\hline HD 146233 & 5818 & 4.45 & 1.00 & 0.04 & 2.22 & 1.03 & 1.57 & 0.07 \\
\hline HD 154962 & 5827 & 4.17 & 1.22 & 0.32 & 4.67 & 1.20 & 2.31 & 0.02 \\
\hline HD 157347 & 5676 & 4.38 & 0.91 & 0.02 & 8.22 & 0.94 & $<0.38$ & - \\
\hline
\end{tabular}


E. Delgado Mena et al.: Li depletion in solar analogues with exoplanets

Table 6. continued

\begin{tabular}{|c|c|c|c|c|c|c|c|c|}
\hline Star & $\begin{array}{l}T_{\text {eff }} \\
(\mathrm{K})\end{array}$ & $\begin{array}{c}\log g \\
\left(\mathrm{~cm} \mathrm{~s}^{-2}\right)\end{array}$ & $\begin{array}{c}\xi_{\mathrm{t}} \\
\left(\mathrm{km} \mathrm{s}^{-1}\right)\end{array}$ & {$[\mathrm{Fe} / \mathrm{H}]$} & $\begin{array}{r}\text { Age } \\
(\mathrm{Gyr}) \\
\end{array}$ & $\begin{array}{l}\text { Mass } \\
\left(M_{\odot}\right)\end{array}$ & $A(\mathrm{Li})$ & Error \\
\hline HD 161612 & 5616 & 4.45 & 0.88 & 0.16 & 2.05 & 1.00 & $<0.45$ & - \\
\hline HD 171665 & 5655 & 4.41 & 0.89 & -0.05 & 4.72 & 0.94 & 1.64 & 0.05 \\
\hline HD 177409 & 5898 & 4.49 & 0.99 & -0.04 & 1.01 & 1.05 & 2.31 & 0.05 \\
\hline HD 177565 & 5627 & 4.39 & 0.91 & 0.08 & 5.60 & 0.96 & $<0.49$ & - \\
\hline HD 177758 & 5862 & 4.41 & 1.11 & -0.58 & 11.74 & 0.90 & 1.68 & 0.10 \\
\hline HD 183658 & 5803 & 4.40 & 1.00 & 0.03 & 5.97 & 1.00 & 1.09 & 0.10 \\
\hline HD 188748 & 5623 & 4.43 & 0.83 & -0.12 & 3.27 & 0.92 & $<0.33$ & - \\
\hline HD 189625 & 5846 & 4.43 & 1.03 & 0.18 & 1.08 & 1.09 & 2.06 & 0.06 \\
\hline HD 190248 & 5604 & 4.26 & 0.99 & 0.33 & 8.74 & 0.98 & $<0.59$ & - \\
\hline HD 195564 & 5676 & 4.03 & 1.11 & 0.06 & 7.51 & 1.08 & 1.97 & 0.05 \\
\hline HD 198075 & 5846 & 4.56 & 0.95 & -0.24 & 2.01 & 0.96 & 1.95 & 0.07 \\
\hline HD 202605 & 5658 & 4.49 & 1.02 & 0.18 & 2.73 & 1.01 & 1.19 & 0.10 \\
\hline HD 203432 & 5645 & 4.39 & 0.98 & 0.29 & 5.81 & 1.00 & $<0.45$ & - \\
\hline HD 206172 & 5608 & 4.49 & 0.77 & -0.24 & 2.94 & 0.89 & 1.27 & 0.10 \\
\hline HD 207700 & 5666 & 4.29 & 0.98 & 0.04 & 10.85 & 0.96 & $<0.42$ & - \\
\hline HD 208704 & 5826 & 4.38 & 1.04 & -0.09 & 9.01 & 0.95 & 1.09 & 0.10 \\
\hline HD 210918 & 5755 & 4.35 & 0.99 & -0.09 & 10.80 & 0.93 & $<0.28$ & - \\
\hline HD 211415 & 5850 & 4.39 & 0.99 & -0.21 & 9.44 & 0.92 & 1.80 & 0.05 \\
\hline HD 212708 & 5681 & 4.35 & 0.99 & 0.27 & 4.77 & 1.02 & $<0.55$ & - \\
\hline HD 213575 & 5671 & 4.18 & 1.02 & -0.15 & 11.65 & 0.95 & $<0.38$ & - \\
\hline HD 214385 & 5654 & 4.43 & 0.81 & -0.34 & 10.64 & 0.82 & $<-0.30$ & - \\
\hline HD 216777 & 5623 & 4.51 & 0.81 & -0.38 & 7.02 & 0.82 & 1.63 & 0.05 \\
\hline HD 220507 & 5698 & 4.29 & 1.01 & 0.01 & 10.62 & 0.96 & $<0.56$ & - \\
\hline HD 221146 & 5876 & 4.27 & 1.09 & 0.08 & 6.43 & 1.09 & $<0.98$ & - \\
\hline HD 221420 & 5847 & 4.03 & 1.28 & 0.33 & 3.76 & 1.29 & 2.65 & 0.02 \\
\hline HD 222595 & 5648 & 4.46 & 0.88 & 0.01 & 1.19 & 0.98 & $<0.58$ & - \\
\hline HD 222669 & 5894 & 4.46 & 1.01 & 0.05 & 1.12 & 1.07 & 2.00 & 0.03 \\
\hline HD 223171 & 5841 & 4.20 & 1.12 & 0.12 & 6.33 & 1.10 & 2.05 & 0.03 \\
\hline HD 224393 & 5774 & 4.54 & 0.84 & -0.38 & 3.73 & 0.87 & 2.21 & 0.04 \\
\hline \multicolumn{9}{|l|}{ HARPS-4 } \\
\hline HD 16784 & 5837 & 4.34 & 1.14 & -0.65 & 11.35 & 0.86 & 1.52 & 0.12 \\
\hline HD 17865 & 5877 & 4.32 & 1.16 & -0.57 & 11.47 & 0.89 & 1.77 & 0.04 \\
\hline HD 22879 & 5884 & 4.52 & 1.20 & -0.81 & 11.38 & 0.81 & 1.63 & 0.05 \\
\hline HD 51754 & 5848 & 4.49 & 1.05 & -0.55 & 8.16 & 0.85 & 1.12 & 0.10 \\
\hline HD 56274 & 5734 & 4.51 & 0.94 & -0.54 & 6.15 & 0.83 & 2.11 & 0.03 \\
\hline HD 69611 & 5762 & 4.31 & 0.99 & -0.58 & 11.31 & 0.85 & $<0.89$ & - \\
\hline HD 75745 & 5885 & 4.29 & 1.34 & -0.78 & 0.00 & 0.00 & 2.42 & 0.04 \\
\hline HD 77110 & 5717 & 4.48 & 0.86 & -0.50 & 7.90 & 0.84 & 1.18 & 0.08 \\
\hline HD 79601 & 5825 & 4.32 & 1.09 & -0.59 & 11.43 & 0.85 & 1.22 & 0.06 \\
\hline HD 88725 & 5654 & 4.49 & 0.86 & -0.64 & 10.71 & 0.80 & $<0.02$ & - \\
\hline HD 97783 & 5682 & 4.50 & 0.88 & -0.73 & 7.75 & 0.79 & $<0.15$ & - \\
\hline HD 104800 & 5697 & 4.47 & 0.87 & -0.79 & 6.34 & 0.78 & $<0.65$ & - \\
\hline HD 105004 & 5756 & 4.33 & 0.80 & -0.81 & 4.30 & 0.79 & 1.86 & 0.05 \\
\hline HD 111777 & 5666 & 4.46 & 0.82 & -0.68 & 8.48 & 0.79 & $<0.44$ & - \\
\hline HD 113679 & 5768 & 4.26 & 1.08 & -0.61 & 6.37 & 0.83 & 2.11 & 0.05 \\
\hline HD 121004 & 5687 & 4.48 & 0.76 & -0.71 & 6.31 & 0.79 & $<0.64$ & - \\
\hline HD 124785 & 5867 & 4.20 & 1.29 & -0.56 & 7.66 & 1.02 & $<0.98$ & - \\
\hline HD 129229 & 5872 & 3.89 & 1.37 & -0.42 & 5.04 & 1.13 & $<0.32$ & - \\
\hline HD 134088 & 5675 & 4.46 & 0.86 & -0.75 & 9.75 & 0.78 & 1.10 & 0.12 \\
\hline HD 134113 & 5782 & 4.25 & 1.27 & -0.74 & 10.92 & 0.88 & 1.90 & 0.05 \\
\hline HD 147518 & 5626 & 4.40 & 0.67 & -0.63 & 6.34 & 0.80 & $<0.54$ & - \\
\hline HD 149747 & 5823 & 3.95 & 1.28 & -0.34 & 0.00 & 0.00 & $<1.48$ & - \\
\hline HD 167300 & 5837 & 4.30 & 1.05 & -0.45 & 10.15 & 0.94 & 1.69 & 0.07 \\
\hline HD 175179 & 5764 & 4.46 & 0.88 & -0.66 & 6.93 & 0.81 & $<0.53$ & - \\
\hline HD 193901 & 5611 & 4.41 & 0.54 & -1.07 & 7.64 & 0.74 & 1.90 & 0.05 \\
\hline HD 197083 & 5735 & 4.50 & 0.90 & -0.45 & 6.62 & 0.85 & 1.38 & 0.06 \\
\hline HD 197197 & 5812 & 4.20 & 1.25 & -0.46 & 10.98 & 0.93 & 2.11 & 0.03 \\
\hline HD 199288 & 5746 & 4.46 & 0.93 & -0.63 & 11.41 & 0.82 & 0.94 & 0.10 \\
\hline HD 199604 & 5817 & 4.34 & 1.04 & -0.62 & 10.63 & 0.83 & 1.46 & 0.10 \\
\hline HD 199847 & 5763 & 4.22 & 1.04 & -0.54 & 9.61 & 0.85 & 1.28 & 0.12 \\
\hline HD 206998 & 5822 & 4.24 & 1.13 & -0.69 & 10.80 & 0.89 & 2.04 & 0.05 \\
\hline HD 224817 & 5894 & 4.36 & 1.13 & -0.53 & 11.08 & 0.91 & 1.80 & 0.04 \\
\hline $\mathrm{BD}+083095$ & 5728 & 4.12 & 0.85 & -0.77 & 0.00 & 0.00 & 2.00 & 0.08 \\
\hline \multicolumn{9}{|l|}{ HARPS-2 } \\
\hline HD 67 & 5746. & 4.56 & 1.02 & 0.03 & 1.73 & 0.98 & 1.91 & 0.05 \\
\hline
\end{tabular}


Table 6. continued.

\begin{tabular}{|c|c|c|c|c|c|c|c|c|}
\hline Star & $\begin{array}{l}T_{\text {eff }} \\
(\mathrm{K})\end{array}$ & $\begin{array}{c}\log g \\
\left(\mathrm{~cm} \mathrm{~s}^{-2}\right) \\
\end{array}$ & $\begin{array}{c}\begin{array}{c}\xi_{\mathrm{t}} \\
\left(\mathrm{km} \mathrm{s}^{-1}\right)\end{array} \\
\end{array}$ & {$[\mathrm{Fe} / \mathrm{H}]$} & $\begin{array}{r}\text { Age } \\
\text { (Gyr) }\end{array}$ & $\begin{array}{l}\text { Mass } \\
\left(M_{\odot}\right) \\
\end{array}$ & $A(\mathrm{Li})$ & Error \\
\hline HD 1979 & 5626. & 4.52 & 0.74 & -0.09 & 4.65 & 0.92 & $<0.80$ & - \\
\hline HD 3220 & 5846. & 4.51 & 0.87 & -0.22 & 3.02 & 0.94 & 2.24 & 0.05 \\
\hline HD 3964 & 5729. & 4.50 & 0.85 & 0.05 & 3.09 & 0.99 & $<1.31$ & - \\
\hline HD 4021 & 5831. & 4.71 & 1.32 & 0.02 & 1.88 & 1.00 & 2.48 & 0.10 \\
\hline HD 8038 & 5694. & 4.45 & 0.88 & 0.15 & 3.52 & 1.01 & $<1.20$ & - \\
\hline HD 8930 & 5687. & 4.52 & 0.79 & -0.23 & 4.27 & 0.90 & 1.75 & 0.10 \\
\hline HD 10895 & 5685. & 4.52 & 0.78 & -0.27 & 5.06 & 0.88 & 1.72 & 0.08 \\
\hline HD 13578 & 5842. & 4.18 & 1.12 & -0.03 & 7.59 & 1.04 & 2.11 & 0.04 \\
\hline HD 14868 & 5864. & 4.44 & 0.90 & 0.02 & 1.87 & 1.02 & 1.71 & 0.10 \\
\hline HD 16008 & 5712. & 4.42 & 0.78 & -0.09 & 5.72 & 0.94 & 1.62 & 0.10 \\
\hline HD 16548 & 5690. & 3.96 & 1.15 & 0.15 & 5.25 & 1.19 & 2.44 & 0.04 \\
\hline HD 17439 & 5721. & 4.44 & 0.87 & 0.08 & 3.05 & 1.00 & $<0.42$ & - \\
\hline HD 18001 & 5772. & 4.44 & 0.80 & -0.07 & 3.05 & 0.96 & 1.28 & 0.10 \\
\hline HD 19423 & 5752. & 4.23 & 0.96 & -0.09 & 10.10 & 0.96 & $<0.20$ & - \\
\hline HD 19641 & 5806. & 4.39 & 0.94 & -0.01 & 4.60 & 0.99 & 1.37 & 0.10 \\
\hline HD 22177 & 5666. & 4.26 & 1.02 & 0.20 & 8.05 & 1.03 & $<0.38$ & - \\
\hline HD 22249 & 5773. & 4.63 & 0.96 & -0.06 & 2.57 & 0.96 & 2.48 & 0.08 \\
\hline HD 25912 & 5900. & 4.52 & 0.99 & 0.12 & 1.37 & 1.06 & 2.15 & 0.05 \\
\hline HD 26729 & 5718. & 4.17 & 1.13 & 0.31 & 6.00 & 1.13 & 1.70 & 0.07 \\
\hline HD 27471 & 5871. & 4.25 & 1.13 & 0.11 & 5.31 & 1.14 & 2.18 & 0.05 \\
\hline HD 29137 & 5768. & 4.28 & 1.10 & 0.30 & 6.29 & 1.08 & $<0.84$ & - \\
\hline HD 29263 & 5780. & 4.35 & 0.94 & 0.03 & 7.02 & 1.00 & $<0.76$ & - \\
\hline HD 29303 & 5819. & 4.52 & 0.93 & -0.12 & 3.24 & 0.97 & 2.23 & 0.08 \\
\hline HD 29428 & 5743. & 4.48 & 0.82 & -0.06 & 2.59 & 0.95 & 1.78 & 0.10 \\
\hline HD 31532 & 5896. & 4.07 & 1.33 & -0.08 & 4.55 & 1.22 & 1.92 & 0.07 \\
\hline HD 33822 & 5726. & 4.29 & 0.98 & 0.26 & 6.79 & 1.05 & $<1.41$ & - \\
\hline HD 34327 & 5883. & 4.20 & 1.19 & -0.06 & 6.51 & 1.11 & 2.46 & 0.06 \\
\hline HD 38078 & 5651. & 4.47 & 0.62 & -0.29 & 5.42 & 0.87 & $<1.31$ & - \\
\hline HD 39427 & 5682. & 4.52 & 0.86 & -0.18 & 4.24 & 0.91 & 1.61 & 0.07 \\
\hline HD 40865 & 5722. & 4.49 & 0.91 & -0.38 & 6.68 & 0.86 & 1.10 & 0.10 \\
\hline HD 41248 & 5713. & 4.49 & 0.84 & -0.37 & 4.99 & 0.86 & 1.56 & 0.10 \\
\hline HD 41323 & 5756. & 4.56 & 0.84 & -0.31 & 4.20 & 0.89 & 1.81 & 0.06 \\
\hline HD 48115 & 5825. & 4.48 & 0.89 & -0.19 & 3.23 & 0.95 & 2.17 & 0.05 \\
\hline HD 49035 & 5640. & 4.35 & 1.01 & 0.24 & 4.07 & 1.00 & $<0.51$ & - \\
\hline HD 61383 & 5716. & 4.20 & 1.12 & -0.49 & 11.41 & 0.92 & 2.04 & 0.05 \\
\hline HD 61447 & 5637. & 4.37 & 0.89 & 0.17 & 3.75 & 0.99 & $<0.89$ & - \\
\hline HD 61986 & 5725. & 4.48 & 0.85 & -0.34 & 5.81 & 0.87 & 1.18 & 0.12 \\
\hline HD 62128 & 5828. & 4.22 & 1.16 & 0.33 & 5.26 & 1.12 & $<0.83$ & - \\
\hline HD 72374 & 5767. & 4.35 & 0.90 & -0.09 & 7.77 & 0.95 & $<0.75$ & - \\
\hline HD 74698 & 5783. & 4.27 & 1.01 & 0.07 & 7.49 & 1.02 & $<0.64$ & - \\
\hline HD 76440 & 5764. & 4.43 & 0.89 & -0.01 & 3.71 & 0.98 & $<0.59$ & - \\
\hline HD 81700 & 5882. & 4.53 & 0.98 & 0.12 & 1.52 & 1.06 & 2.18 & 0.05 \\
\hline HD 87320 & 5639. & 4.41 & 0.95 & -0.17 & 9.27 & 0.90 & $<0.88$ & - \\
\hline HD 90702 & 5760. & 4.56 & 1.04 & 0.20 & 1.96 & 1.04 & 1.92 & 0.08 \\
\hline HD 90722 & 5711. & 4.28 & 1.06 & 0.31 & 6.58 & 1.03 & $<0.37$ & - \\
\hline HD 91345 & 5658. & 4.52 & 0.72 & -1.04 & 8.73 & 0.75 & 1.61 & 0.10 \\
\hline HD 94771 & 5631. & 4.03 & 1.10 & 0.22 & 6.83 & 1.11 & 1.52 & 0.08 \\
\hline HD 96116 & 5832. & 4.52 & 0.96 & -0.01 & 2.74 & 1.00 & 2.12 & 0.07 \\
\hline HD 101339 & 5731. & 4.48 & 0.88 & -0.10 & 4.26 & 0.95 & 1.21 & 0.10 \\
\hline HD 101367 & 5615. & 4.38 & 0.91 & 0.29 & 7.30 & 0.99 & $<0.82$ & - \\
\hline HD 101644 & 5678. & 4.58 & 0.72 & -0.56 & 5.04 & 0.82 & 2.05 & 0.05 \\
\hline HD 105779 & 5792. & 4.51 & 0.91 & -0.25 & 4.05 & 0.92 & 1.63 & 0.08 \\
\hline HD 108768 & 5633. & 4.45 & 0.86 & 0.14 & 2.80 & 0.98 & $<0.79$ & - \\
\hline HD 109098 & 5888. & 4.14 & 1.24 & 0.06 & 5.42 & 1.14 & 2.48 & 0.05 \\
\hline HD 109723 & 5647. & 4.47 & 0.88 & -0.04 & 3.18 & 0.93 & 1.58 & 0.20 \\
\hline HD 110668 & 5850. & 4.45 & 0.98 & 0.17 & 2.22 & 1.07 & 1.60 & 0.15 \\
\hline HD 113513 & 5751. & 4.54 & 0.94 & 0.17 & 1.49 & 1.01 & 1.88 & 0.20 \\
\hline HD 114561 & 5829. & 4.50 & 0.88 & -0.07 & 2.97 & 0.99 & $<1.10$ & - \\
\hline HD 115902 & 5705. & 4.39 & 0.86 & -0.01 & 4.36 & 0.96 & $<0.74$ & - \\
\hline HD 116259 & 5700. & 4.21 & 0.99 & 0.11 & 8.56 & 1.02 & $<0.45$ & - \\
\hline HD 119173 & 5779. & 4.26 & 0.52 & -0.62 & 5.66 & 0.82 & 1.94 & 0.10 \\
\hline HD 120344 & 5623. & 4.42 & 0.65 & -0.19 & 5.24 & 0.90 & $<0.42$ & - \\
\hline HD 122474 & 5716. & 4.37 & 0.88 & 0.13 & 3.48 & 1.01 & $<0.77$ & - \\
\hline HD 123319 & 5619. & 4.56 & 0.67 & -0.52 & 4.45 & 0.81 & 2.31 & 0.03 \\
\hline HD 129191 & 5832. & 4.39 & 0.99 & 0.24 & 2.49 & 1.08 & 1.12 & 0.10 \\
\hline
\end{tabular}


E. Delgado Mena et al.: Li depletion in solar analogues with exoplanets

Table 6. continued.

\begin{tabular}{|c|c|c|c|c|c|c|c|c|}
\hline Star & $\begin{array}{l}T_{\text {eff }} \\
(\mathrm{K})\end{array}$ & $\begin{array}{c}\log g \\
\left(\mathrm{~cm} \mathrm{~s}^{-2}\right)\end{array}$ & $\begin{array}{c}\xi_{\mathrm{t}} \\
\left(\mathrm{km} \mathrm{s}^{-1}\right)\end{array}$ & {$[\mathrm{Fe} / \mathrm{H}]$} & $\begin{array}{l}\text { Age } \\
(\mathrm{Gyr})\end{array}$ & $\begin{array}{l}\text { Mass } \\
\left(M_{\odot}\right)\end{array}$ & $A(\mathrm{Li})$ & Error \\
\hline HD 131183 & 5670. & 4.24 & 0.97 & 0.09 & 9.36 & 0.99 & $<0.33$ & - \\
\hline HD 131218 & 5797. & 4.55 & 0.96 & -0.06 & 2.48 & 0.97 & 2.18 & 0.07 \\
\hline HD 131565 & 5612. & 4.47 & 0.72 & -0.16 & 5.84 & 0.90 & $<1.23$ & - \\
\hline HD 134702 & 5782. & 4.50 & 0.74 & -0.04 & 3.20 & 0.98 & 1.98 & 0.08 \\
\hline HD 140785 & 5756. & 4.12 & 1.09 & -0.05 & 7.28 & 1.09 & 2.08 & 0.05 \\
\hline HD 141624 & 5871. & 4.40 & 1.01 & -0.38 & 8.59 & 0.88 & 1.61 & 0.15 \\
\hline HD 142879 & 5707. & 4.51 & 0.74 & -0.39 & 5.03 & 0.85 & 1.84 & 0.08 \\
\hline HD 145927 & 5819. & 4.41 & 0.93 & -0.03 & 2.74 & 0.99 & $<0.46$ & - \\
\hline HD 148577 & 5713. & 4.29 & 0.95 & -0.09 & 10.46 & 0.96 & $<0.16$ & - \\
\hline HD 149396 & 5657. & 4.47 & 0.91 & 0.19 & 4.95 & 1.00 & $<0.71$ & - \\
\hline HD 149724 & 5747. & 4.24 & 1.08 & 0.37 & 6.57 & 1.07 & 2.08 & 0.06 \\
\hline HD 150437 & 5826. & 4.29 & 1.09 & 0.30 & 4.63 & 1.08 & 1.46 & 0.15 \\
\hline HD 151933 & 5849. & 4.29 & 1.03 & -0.21 & 5.32 & 0.94 & 1.61 & 0.07 \\
\hline HD 155968 & 5790. & 4.42 & 0.92 & 0.16 & 2.46 & 1.04 & $<0.90$ & - \\
\hline HD 156079 & 5892. & 4.24 & 1.16 & 0.28 & 4.29 & 1.21 & 2.27 & 0.04 \\
\hline HD 161256 & 5652. & 4.27 & 0.93 & 0.14 & 6.97 & 0.99 & $<0.18$ & - \\
\hline HD 161555 & 5850. & 4.15 & 1.23 & 0.12 & 5.14 & 1.16 & 2.64 & 0.06 \\
\hline HD 165131 & 5870. & 4.45 & 0.99 & 0.06 & 2.43 & 1.04 & 1.53 & 0.10 \\
\hline HD 166745 & 5621. & 4.31 & 0.98 & 0.25 & 5.06 & 1.00 & $<0.15$ & - \\
\hline HD 171942 & 5615. & 4.42 & 0.72 & -0.10 & 4.54 & 0.92 & $<-0.33$ & - \\
\hline HD 172568 & 5728. & 4.58 & 0.78 & -0.37 & 5.48 & 0.86 & $<0.38$ & - \\
\hline HD 172643 & 5645. & 4.50 & 0.73 & -0.15 & 3.51 & 0.90 & 1.29 & 0.20 \\
\hline HD 178904 & 5727. & 4.41 & 0.98 & 0.09 & 6.10 & 1.00 & $<0.52$ & - \\
\hline HD 186302 & 5662. & 4.44 & 0.78 & -0.03 & 3.63 & 0.95 & $<-0.29$ & - \\
\hline HD 190524 & 5825. & 4.50 & 0.85 & -0.13 & 4.21 & 0.96 & 2.10 & 0.07 \\
\hline HD 190613 & 5776. & 4.33 & 0.95 & 0.00 & 7.14 & 0.98 & 1.31 & 0.15 \\
\hline HD 195145 & 5625. & 4.41 & 0.82 & 0.17 & 3.61 & 0.99 & $<0.28$ & - \\
\hline HD 200633 & 5853. & 4.51 & 1.01 & 0.05 & 2.41 & 1.03 & $<1.27$ & - \\
\hline HD 201422 & 5841. & 4.63 & 0.98 & -0.16 & 2.82 & 0.96 & 2.45 & 0.07 \\
\hline HD 204287 & 5743. & 4.15 & 1.10 & -0.04 & 8.73 & 1.03 & 1.52 & 0.05 \\
\hline HD 209449 & 5854. & 4.16 & 1.19 & 0.41 & 4.69 & 1.18 & 2.32 & 0.05 \\
\hline HD 210272 & 5713. & 4.13 & 1.16 & -0.22 & 8.53 & 1.04 & 2.02 & 0.05 \\
\hline HD 212036 & 5687. & 4.41 & 0.80 & -0.01 & 5.05 & 0.96 & $<0.04$ & - \\
\hline HD 212231 & 5762. & 4.20 & 1.01 & -0.30 & 11.06 & 0.92 & $<1.00$ & - \\
\hline HD 214867 & 5807. & 4.66 & 1.23 & 0.01 & 1.82 & 0.99 & 2.41 & 0.08 \\
\hline HD 214954 & 5738. & 4.48 & 0.99 & 0.14 & 4.83 & 1.02 & $<0.77$ & - \\
\hline HD 216008 & 5773. & 4.38 & 0.91 & -0.04 & 6.13 & 0.97 & $<0.52$ & - \\
\hline HD 218340 & 5889. & 4.42 & 0.97 & 0.09 & 2.05 & 1.06 & 2.07 & 0.05 \\
\hline HD 218885 & 5763. & 4.46 & 0.80 & -0.28 & 3.26 & 0.89 & 2.01 & 0.10 \\
\hline HD 220456 & 5887. & 4.50 & 1.01 & -0.02 & 2.00 & 1.02 & 2.26 & 0.05 \\
\hline HD 221343 & 5848. & 4.54 & 0.99 & 0.11 & 1.56 & 1.04 & 1.95 & 0.07 \\
\hline HD 223315 & 5650. & 4.44 & 0.89 & 0.30 & 1.79 & 1.00 & $<1.03$ & - \\
\hline HIP 99606B & 5764. & 4.19 & 0.98 & -0.41 & 3.55 & 0.86 & 1.68 & 0.10 \\
\hline
\end{tabular}

